\title{
Association of Metabolic Syndrome and
}

Cardiovascular Risk Factors Among Colorectal

Cancer Patients Attending in Tikur Anbessa

Specialized Hospital Oncology Units: A Comparative

Cross-sectional Study June, 2017 Addis Ababa,

Ethiopia

Shimelis Adugna Elemo ( $\nabla$ shimeadu39@gmail.com )

Department of Medical Laboratory Sciences, Collage of Health Sciences, Arsi University,P.O. Box 396, Asella, Ethiopia, https://orcid.org/0000-0002-7371-1220

Bereket Mihretu Awoke

Debremarkos University

Research article

Keywords: colon cancer, rectal cancer, metabolic syndrome, obesity

Posted Date: October 5th, 2020

DOI: https://doi.org/10.21203/rs.3.rs-73476/v1

License: (c) (1) This work is licensed under a Creative Commons Attribution 4.0 International License.

Read Full License 
Association of Metabolic Syndrome and Cardiovascular Risk factors Among Colorectal Cancer Patients Attending in Tikur Anbessa Specialized Hospital Oncology Units: A comparative crosssectional study June, 2017 Addis Ababa, Ethiopia

Authors:

1. Bereket Mihretu Awoke ${ }^{a}$

2. Shimeles Adugna Elemo ${ }^{b^{*}}$

${ }^{a}$ Department of Biomedical Sciences, Collage of Health Sciences, Debremarkos University

P.O. Box 1176, Debremarkos, Ethiopia E.mail: mihretubereket@gmail.com

${ }^{\mathrm{d}}$ Department of Medical Laboratory Sciences, Collage of Health Sciences, Arsi

University,P.O. Box 396, Asella, Ethiopia, E-mail: Shimeadu39@gmail.com

Corresponding author: Shimeles Adugna Elemo, E-mail: Shimeadu39@gmail.com

\section{Abstract}

Background: Metabolic syndrome and obesity are risk factors for developing type 2 diabetes mellitus and/or cardiovascular disease, especially stroke. Obesity-associated diseases account for a large portion of public health challenges. Among obesity-related disorders, a direct and independent relationship has been ascertained for colorectal cancer.

Objective: The aim of this study was to determine the prevalence of metabolic syndrome and other cardiovascular risk factors, including dyslipidemia, hypertension, dietary and exercise practices, tobacco use and body mass index in individual with colorectal cancer patients attending Tikur Anbessa Hospital Oncology department, Addis Ababa.

Methods: A comparative cross-sectional study was conducted from February, 2016 to Jun 2017 on 79 patients attending the Oncology clinic at Tikur Anbessa Hospital. Components of metabolic syndrome, including fasting serum glucose, serum triglyceride, serum high density lipoprotein, blood pressure and waist circumference, was measured in all of the patients. In addition, serum total cholesterol, serum low density lipoprotein was measured with 5010 
photometers, and information gathered on smoking and other lifestyle practices, including diet and exercise.

Result: Of 79 patients, 23 had metabolic syndrome (MS); 58.2\% males; mean-age 48.6 years with range (26 to 78 ) and $41.8 \%$ Female; mean age 44.6 years with range (20 to 78 ). Among the MS group, $45.6 \%$ had high blood pressure, $26.6 \%$ had increased waist circumference, $67.1 \%$ had serum blood glucose level greater than $100 \mathrm{mg} / \mathrm{dL}, 68.3 \%$ had low high-density lipoprotein cholesterol level, $57 \%$ had increased triglyceride level, and $3.8 \%$ were obese [body mass index $(\mathrm{BMI})>30 \mathrm{~kg} / \mathrm{m} 2$ ]. $29 \%$ presented 3 criteria of MS. From total colorectal cancer patients $53.2 \%$ had colon cancer, $46.8 \%$ had rectal cancer and $12.7 \%$ had stage I,40.5 \% had stage II (IIa,IIb \& IIc), $25.3 \%$ had stage III (a,b,c) and $21.5 \%$ had stage IV disease.

CONCLUSION: MS is positively associated with adenomas and colorectal cancer. However, there is not enough information in Ethiopia to justify screening in patients with MS. To our knowledge, no previous study has evaluated this association in Ethiopian patients.

KEYWORDS: colon cancer, rectal cancer, metabolic syndrome, obesity

\section{Background}

\section{Definition}

Colorectal cancer is a term used to refer to cancer that develops in the colon or the rectum. These cancers are sometimes called colon cancer or rectal cancer, depending on where they start. Colon cancer and rectal cancer are often grouped together because they have many features in common [1]. Colorectal cancer begins when healthy cells in the lining of the colon or rectum change and grow uncontrollably, forming a mass called a tumor. A tumor can be cancerous or benign. A cancerous tumor is malignant, meaning it can grow and spread to other parts of the body [2]. A benign tumor means the tumor can grow but will not spread. These changes usually take years to develop. However, when a person has an uncommon inherited syndrome changes can occur within months to years. Both genetic and environmental factors can cause the changes [3]. 


\section{Anatomy of the colon and rectum}

The colon and rectum make up the large intestine, which plays an important role in the body's ability to process waste. The colon makes up the first five to six feet of the large intestine, and the rectum makes up the last six inches, ending at the anus. The colon has four sections. The ascending colon is the portion that extends from a pouch called the cecum, the beginning of the large intestine into which the small intestine empties, on the right side of the abdomen. The transverse colon crosses the top of the abdomen. The descending colon takes waste down the left side. Finally, the sigmoid colon at the bottom takes waste a few more inches, down to the rectum. Waste leaves the body through the anus $[3,4]$.

\section{Epidemiology of Colorectal Cancer}

Colorectal cancer incidence and mortality rates vary markedly around the world. Globally, colorectal cancer is the third most commonly diagnosed cancer in males and the second in females, with over 1.2 million new cases and 608,700 deaths estimated to have occurred in 2008. Rates are substantially higher in males than in females [5].Understanding risk factors for colorectal cancer may guide the development of strategies targeted at its prevention [6].

Colorectal cancer is the third most common incident cancer in the United States, with an estimated 146,940 diagnoses in 2004 , and it is the third leading cause of cancer mortality there. The incidence and mortality have been slowly but steadily decreasing, with a current annual incidence of approximately 132,700 new cases, of which 93,090 are colon and the remainder

rectal cancer. Annually, approximately 49,700 Americans die of colorectal cancer, accounting for approximately 8 percent of all cancer deaths $[7,8]$. 
Colorectal cancer is an important health problem since one million new cases are diagnosed world-wide each year with half million related deaths. The incidence rate of colon cancer according to Five Continents cancer registries varies from 3\% in Africa (Algeria) up to $40 \%$ in North America. In Europe the incidence of colon cancer ranges from 12.1\% in Belarus up to $30.5 \%$ in Italy [9].

Globally, the incidence of colorectal cancer varies over 10 -fold. The highest incidence rates are in Australia and New Zealand, Europe and North America, and the lowest rates are found in Africa and South-Central Asia [5]. These geographic differences appear to be attributable to differences in dietary and environmental exposures that are imposed upon a background of genetically determined susceptibility. Colorectal carcinoma was once thought to be rare in Africans, but is being seen more frequently. Diet and lifestyle modify the risk of colorectal cancer. Its frequency, age, sex and site distribution have not been studied systematically in Ethiopia.

In one study in Ethiopia, colorectal cancer was studied and compared in two 5-year periods with a 10 year time gap. The biopsies of 255 patients with a diagnosis of colorectal cancer during two periods were reviewed. Colorectal cancer constituted $0.8 \%$ of the total of biopsies and $34 \%$ of colorectal biopsies. The mean age at presentation was 47 years old $[10,11]$, while $61.4 \%$ occurred below the age of 50 years, $36 \%$ below 40 years old and $16 \%$ occurred below the age of 30 years. Of all colorectal cancers, $66.7 \%$ were located in the rectum and $33.3 \%$ in the colon. The male to female ratio for both rectal and colonic cancers was 2:1. These findings did not show any major change during the two study periods. Colorectal cancer therefore occurs at a much younger age in Ethiopia than in the developed world and is more commonly rectal cancer. Therefore, the shift of colorectal cancer to the right colon in recent years that has been reported 4| P a g e 
elsewhere was not observed in this Ethiopian study. The clinician should expect colorectal cancer also to occur more frequently in young patients, and most of these carcinomas are still detectable by proctosigmoidoscopy [11].

\section{Etiology of Colorectal Cancer}

Two well-defined hereditary syndromes account for around 5 to $10 \%$ of colorectal cancers: familial adenomatous polyposis (FAP) and hereditary non polyposis colorectal cancer (HNPCC). FAP is caused by mutations of the APC gene and HNPCC is caused by mutations in the MMR gene. Around $5 \%$ of colorectal cancers are estimated to be attributable to APC and MMR mutations, and up to $20 \%$ of colorectal cancers are familial [12].

Ulcerative colitis and Crohn's disease are inflammatory bowel diseases that have been related to an increased risk of colorectal cancer. Persons with ulcerative colitis have an approximately 1.1 to 2.8-fold increased risk of colorectal cancer, and risk increases with duration of the disease. Crohn's disease has been associated with and approximately 2 -fold increased risk. The use of non-steroidal anti-inflammatory drugs (NSAIDs) has been shown to reduce the risk of colorectal cancer [13].

Insulin-like growth factor-I (IGF-I) is a growth stimulating hormone which peaks in puberty and declines thereafter. In vitro and in vivo studies have further shown that IGF-I stimulates tumour growth, which has raised an interest to further study the association with cancer. Bioactivity of IGF-I is regulated by IGF-binding proteins (IGFBP). Only $1 \%$ of IGF-I circulates in its free form, and $99 \%$ of IGF-I is bound to IGFBPs, of which IGFBP-3 is the most common type. Free IGF-I - the more potent form of IGF-I - has rarely been studied on the association with cancer risk, but total IGF-I, and also IGFBP-3, is strongly related to free IGF-I. Elevated IGF-I has been 
related to a significant increased risk of colorectal cancer, and IGFBP-3 has overall not been related to risk [14].

Compared to other cancers of the gastrointestinal tract, the association between tobacco smoking and risk of colorectal cancer is modest. However, a recent meta-analysis showed that current and ex-smokers had a significant 15 to $20 \%$ higher risk of colorectal cancer than never smokers and the risk increased with duration and dose of smoking. Lifestyle factors, including exercise and diet, are also involved in the etiology of colorectal cancer [12].

\section{Mutation on APC gene in colonic epithelial cells}

In normal colon epithelial cells, secreted frizzled-related proteins (SFRPs) function as antagonists of wnt (integration and wingless gene) signaling by competing with Wnt proteins for binding to their receptor, Frizzled (FRZ). Expression of Sfrps is therefore the epigenetic gatekeeper step. When wnt signaling is inactive, the adenomatosis polyposis coli (APC) complex phosphorylates $\beta$-catenin, leading to its degradation. This prevents the accumulation of nuclear $\beta$-catenin and therefore its ability to engage its transcription factor partners (TGF), which results in the differentiation and homeostasis of colon epithelial cells [15].

Expression of $a p c$ is therefore a genetic gatekeeper step. When sfrp expression is lost, through epigenetic silencing of the gene that encodes it (loss of the epigenetic gatekeeper), wnt signaling becomes activated through the receptor FRZ. This wnt signaling potentially inactivates the APC complex (loss of the genetic gatekeeper), allowing $\beta$-catenin to accumulate in the cytoplasm and eventually in the nucleus. In the nucleus, $\beta$-catenin activates transcription of genes such as $m y c$, cyclin $D$ and other genes whose products promote cell proliferation and survival rather than differentiation. 
This results in the expansion of colon epithelial stem and progenitor cells and formation of aberrant cryptic foci (ACF) $[16,17]$ Persistent activation of the Wnt pathway allows mutations to occur in other pathway components, such as those that permanently disable the APC complex and promote nuclear accumulation of $\beta$-catenin). These cells are selected for because of their survival and proliferative advantages. This combination of epigenetic and genetic events fully activates the Wnt pathway to promote tumor progression [18]. Without the epigenetic events that silence the sfrp genes, mutations that disrupt the APC complex might not be sufficient to promote tumorigenesis or tumor progression [2].

\section{Clinical stage of colorectal cancer}

Staging of colorectal cancer refers to how far a cancer has spread on a scale from 0 to IV, with Stage 0 meaning the cancer that has not begun to invade the colon wall and Stage IV describing cancer that has spread beyond the original site to other parts of the body. Tumors are staged or graded for severity, according to evidence of invasion into the intestinal wall, or evidence of more distant spread. There is a close correlation between advancing stage and cancer mortality. Tumor size does not appear to be important in terms of outcome. The aggressiveness of colorectal cancer is based upon its ability to grow and invade the colonic wall, lymphatic system, and blood vessels [19].

\section{Classification of Tumor Spread}

Two staging systems are used to define the extent of invasion of colorectal cancer: the Dukes' classification and the TNM staging system (table 1). 
In both systems, carcinoma in situ (also referred to as high grade dysplasia) is defined as intramucosal carcinoma that does not penetrate the muscularis mucosae [20].

\section{TNM Staging System}

The TNM system, developed by the American Joint Committee on Cancer (AJCC) is the most widely used, and is considered the most precise and descriptive. T stands for tumor and the depth to which it has penetrated the colon wall, $\mathbf{N}$ stands for lymph node involvement, and $\mathrm{M}$ refers to metastases, or whether the cancer has spread to other body parts [21, 22].

\section{Metabolic Syndrome and Colorectal Cancer}

Definition: - Metabolic syndrome is a constellation of interrelated risk factors of metabolic origin (metabolic risk factors) that appear to directly promote the development of atherosclerotic cardiovascular disease. The primary components of metabolic syndrome include insulin resistance, abdominal obesity, dyslipidemia, and hypertension but with continuing emergence of new facts, the list tends to be growing. The components therefore now include insulin resistance, hyperinsulinemia, central obesity, hypertension, dyslipidemia [increase in plasma triglycerides (TG), decrease in high density lipoprotein cholesterol (HDL-C) and an LDL particle pattern shifted to small dense particles (type B pattern)], procoagulant state (increased plasma fibrinogen, increased plasminogen activator inhibitor-1 (PAI-1)), fatty liver, vascular abnormalities (increase in urinary albumin excretion, endothelial dysfunction), inflammatory markers, and hyperuricemia [23].

Metabolic syndrome is defined in this thesis as the occurrence of any three of the five components, based on the criteria specified by Adult Treatment Panel III ( ATP III): (1) Abdominal obesity: waist circumference $\geq 102 \mathrm{~cm}$ in men and $\geq 88 \mathrm{~cm}$ in women; (2) Elevated 
triglyceride (TG > $150 \mathrm{mg} / \mathrm{dl}$ ) in both men and women (or if a person is taking medication for dyslipidemia); (3) low HDL-C, with an HDL-C value $<40 \mathrm{mg} / \mathrm{dL}$ in men and $<50 \mathrm{mg} / \mathrm{dL}$ in women; (4) Elevated blood pressure (BP): systolic BP $\geq 130 \mathrm{mmHg}$ and/or diastolic $\mathrm{BP} \geq 85$ $\mathrm{mmHg}$ (or if a person is taking medication for hypertension); (5) Elevated fasting serum glucose above $6.1 \mathrm{mmol} / \mathrm{l}$ or $100 \mathrm{mg} / \mathrm{dL}$ in both men and women (or if a person is taking medication for diabetes [24-26] .

Several molecular mechanisms have been proposed regarding higher risk of colorectal neoplasm in those with metabolic syndrome including the role of oxidative stress, insulin growth factor-1 (IGF-1), and inflammatory cytokines; all of which are increased in those with metabolic syndrome. Higher levels of reactive oxygen species found in those with metabolic syndrome might lead to DNA damage and thus place subjects at risk for colorectal neoplasm development. IGF-1 has been shown to increase cellular turnover and inhibits apoptosis [24]. It also leads to increased production of vascular endothelial growth factor which supports tumor growth. In individuals with metabolic syndrome, hyper insulinemia either due to obesity or impaired fasting glucose leads to increase in IGF-1 and possibly increases the carcinogenic effects. These effects of IGF-1 have been studied selectively on colonic mucosa in many in vitro studies and their implications were evident in many clinical studies looking at colon cancer risk in diabetes patients [5].

There is evidence that body composition and hormonal factors contribute to colorectal cancer etiology. Excess body mass and abdominal obesity have been associated positively with colorectal cancer in prospective human research. Abdominal obesity may be a stronger risk factor than generalized obesity. Weight gain in middle age that results in abdominal obesity increases the risk of colonic adenomas and colorectal carcinoma. Abdominal obesity is 
associated with hyper insulinemia; in vitro, animal, and human epidemiologic studies support a relation between insulin and colorectal carcinogenesis. Hyper insulinemia and other markers of insulin resistance (such as hyper triglyceridemia or hyperglycemia), diabetes, obesity, and other factors associated with obesity, such as elevated levels of inflammatory factors, growth hormones, and gender hormones, all have been hypothesized as factors that increase the risk of colorectal cancer $[9,27]$.

Baseline metabolic syndrome ( 3 components vs. 0 components) had a positive association with age-adjusted and gender-adjusted colorectal cancer incidence (relative risk [RR], 1.49; 95\% confidence interval $[95 \% \mathrm{CI}], 1.0-2.4)$; this association was attenuated after multivariate adjustment ( $\mathrm{RR}, 1.39$; 95\% CI, 0.9-2.2).There was a dose-response association between colorectal cancer incidence and the number of metabolic syndrome components present at baseline (P. value .006) after multivariate adjustment. Analysis of gender revealed that the multivariate-adjusted association of metabolic syndrome with colorectal cancer was stronger in men (RR, 1.78; 95\%CI, 1.0-3.6) and weaker in women (RR, 1.16; 95\% CI, 0.6-2.2; [28].

Recent findings on the association between metabolic syndrome and the development of colorectal malignancies in western European countries were 258 patients taken of that, 129 had metabolic syndrome; 51\% males; mean-age 67.1 years. Among the Metabolic syndrome group, $94 \%$ had high blood pressure, $91 \%$ had increased waist circumference, $60 \%$ had diabetes, $55 \%$ had low high-density lipoprotein cholesterol level, 50\% had increased triglyceride level, and $54 \%$ were obese [body mass index (BMI) $30 \mathrm{~kg} / \mathrm{m} 2$ ]. $51 \%$ presented 3 criteria of metabolic syndrome. Metabolic syndrome was associated with increased prevalence of adenomas (43\% vs $25 \%, \mathrm{p}=0.004)$ and colorectal cancer $(13 \%$ vs $5 \%, \mathrm{p}=0.027)$, compared with patients without 
metabolic syndrome. Metabolic syndrome was also positively associated with multiple ( $\geq 3$ ) adenomas $(35 \%$ vs $9 \%, \mathrm{p}=0.024)$ and sessile adenomas $(69 \%$ vs $53 \%, \mathrm{p}=0.05)$. No difference existed between location $(\mathrm{p}=0.086)$, grade of dysplasia $(\mathrm{p}=0.196)$, or size $(\mathrm{p}=0.841)$ of adenomas. In addition, no difference was found between Body mass index $(\mathrm{p}=0.078)$, smoking $(\mathrm{p}=0.146)$, alcohol consumption $(\mathrm{p}=0.231)$, and the presence of adenomas [29].

These hypothesized risk factors may interact to increase risk of developing colorectal cancer; therefore, a collection of metabolic factors may be an appropriate model in which to examine colorectal cancer risk. The metabolic syndrome comprises several risk factors that predispose for cardiovascular disease and type 2diabetes and all-cause mortality; it also is hypothesized that the metabolic syndrome increases the risk of colorectal cancer. The prevalence of the metabolic syndrome has been estimated at $24 \%$ and $23 \%$ among adult males and females, respectively, in the United States [30].

Cancer-related mortality is reported to be high in cases with metabolic syndrome. In a 14-year follow-up of 33,230 male cases (28\% with metabolic syndrome) aged between 20 and 88 with no known cancer at baseline, patients with Metabolic syndromes were associated with a $56 \%$ greater age-adjusted risk in cancer mortality. Mortality risk was $83 \%$ higher in individuals with 3 or more metabolic syndrome components than those with none. In the 'Risk Factors and Life Expectancy Project' study, 21,311 male and 15,991 women were followed for an average period of 7 years. Results showed that a cluster of Metabolic Syndrome components has increased colorectal cancer mortality significantly (hazard ratio (HR) 2.96 (1.05-8.31) for male, HR 2.71 (0.59-12.50) for female, HR 2.99 (1.27- 7.01) combined) [31]. 
A cross sectional study was conducted on 300 hypertensive individuals who received follow-up care at University of Gondar Hospital after being diagnosed as hypertensive. The WHO STEPwise approach to surveillance of NCD was used. The prevalence of metabolic syndrome was about $40 \%$ according to the modified NCEP-ATP III and IDF criteria respectively. Low HDL-c was found to be the most frequently encountered (81.3\%) component of metabolic syndrome, other than the hypertension. Being female was significantly associated with metabolic syndrome $(\mathrm{AOR}=4.34 ; 95 \% \mathrm{CI}: 2.09,8.99)$ using $\mathrm{IDF}$ and $(\mathrm{AOR}=3.30 ; 95 \% \mathrm{CI}: 1.66,6.58)$ using NCEP-ATP III criteria. Abnormal BMI which included both high and low BMI was found to have significant association with metabolic syndrome (AOR $=3.10 ; 95 \%$ CI: $1.73,5.58)$ using IDF and (AOR $=1.84 ; 95 \%$ CI: $1.05,3.22)$ as diagnosed using the NCEP-ATP III criteria [32].

\section{Pathophysiological Links between Metabolic Syndrome and Colorectal Cancer}

It has been hypothesized that insulin resistance is the most important underlying mechanism of the metabolic syndrome in close relationship to abdominal obesity. Insulin has been shown to affect growth of normal and neoplastic epithelial cells and to have mitogenic actions in vitro and in experimental models, either directly or indirectly through IGF-1. At high concentrations, insulin can bind to IGF-1 receptors (IGF1Rs) or can act directly to promote IGF-1 biosynthesis, enhancing IGF-1 bioavailability and inhibiting the production of IGFBP-1, IGFBP-2 and IGFBP3. [33].

IGF-1 is an important mitogen required for the progression through the cell cycle and has autocrine, paracrine and endocrine actions on cell proliferation and apoptosis, increasing the risk of cellular transformation by enhancing cell turnover. In addition, IGF-1 increases the production of vascular endothelial growth factor (VEGF), an angiogenic factor that can support cancer 
growth. It has been shown that normal colorectal epithelia and colon cancer cells have both insulin and IGF1Rs [27, 34]). Tissue homeostasis in the normal colonic crypt relies on a balance between proliferation, differentiation and apoptosis, with apoptosis occurring at the top of the colonic crypt as the culmination of a differentiation pathway [9, 35].

The link between IGF-1 and IGFBP-3 levels and the increased risk of colorectal adenoma and cancer came first to attention in acromegalic patients, characterized by chronically elevated growth hormone $(\mathrm{GH})$ levels. GH excess leads to hepatic and peripheral insulin resistance and thus to hyper insulinemia, a common feature of acromegaly and metabolic syndrome, that causes IGF-1 hyper secretion and low IGFBP-3 levels [29].

The relationship between IGF-1 and IGFBP-3 levels and colorectal cancer was examined on 32,826 women from Nurses' Health Study. Controlling for IGFBP-3 level, relative to women in the lowest tertile of IGF-1, those in the highest tertile were at elevated risk of intermediate/latestage colorectal neoplasia adenoma (MVRR: $2.78,95 \%$ CI: 0.76-9.76) and cancer (RR: 2.18, 95\% CI: 0.94-5.08). Controlling for IGF-1 level, relative to women in the lowest tertile of IGFBP-3, women in the highest tertile of IGFBP-3 were at lower risk of intermediate/late-stage colorectal adenoma (RR: $0.28,95 \%$ CI: $0.09-0.85$ ) and cancer (RR: $0.28,95 \%$ CI: $0.10-0.83$ ). Neither IGF-1 nor IGFBP-3 had any appreciable relation with early-stage adenoma. These analyses indicate that high levels of circulating IGF-1 and particularly low levels of IGFBP-3 are associated independently with an elevated risk of large or tubulo-villous/villous colorectal adenoma and cancer. These results are concordant with those obtained previously in Physicians' Health Study [14] 
The role of IGFBP-3 in colorectal cancer was independently analyzed by IGFBP-3 has been shown to enhance p53-dependent apoptosis after DNA damage. Therefore, loss of IGFBP-3 could contribute to the development of colonic adenomas that retain wild-type p53 function through suppression of p53-dependent apoptotic signals, allowing aberrant cell survival and tumor formation [33]. Furthermore there is disruption in both adenoma and carcinoma tissue. This pattern is similar to that of TGF- $\beta$ distribution in normal, adenoma and carcinoma tissue. Because it is known that TGF- $\beta$ is a potent growth inhibitor for colonic epithelium, this similarity suggests that IGFBP-3 may have an important role in the regulation of differentiation and apoptosis in human colonic epithelium $[9,35]$.

The role of insulin resistance and hyperinsulinemia in colorectal cancer was directly assessed by in a study performed on 5849 participants in the Cardiovascular Health Study cohort. The authors identified 102 cases of colorectal cancer. Fasting insulin was not related to an increased risk $(\mathrm{RR}=1.2)$, whereas $2 \mathrm{~h}$ post-meal insulin was related to a significantly increased risk $(\mathrm{RR}=$ 2.0) [35].

\section{Adipokines and Inflammatory Cytokines}

Adipose tissue is a complex endocrine organ, responsible for the secretion and synthesis of hormones, cytokines and other signaling proteins, collectively termed as adipokines. Adipokines are a diverse group of signaling molecules that play roles in such processes as appetite and energy balance, inflammation, insulin resistance/ sensitivity, angiogenesis, lipid metabolism, cell proliferation and atherosclerosis. Many of these functions are related to either the metabolic syndrome or cancer, and they may serve as a link between these two pathologies $[14,36]$. 
Adolescent obesity and inflammation have been associated with subsequent development of colorectal cancer. In one prospective study, adolescent body mass index, and inflammation (as measured by erythrocyte sedimentation rate, ESR), were related to colorectal cancer risk as reported in a national cancer registry. A cohort study was conducted on 239,658 male conscripts, aged 16 to 20 in which body mass index and ESR was measured between1969 to1976. These conscripts were followed for colorectal cancer (CRC) over 35 years of follow up. During this time, $885(0.37 \%)$ cases of colorectal cancer occurred, including $501(0.21 \%)$ colon cancer and $384(0.16 \%)$ rectal cancers [37]. This study provided convincing evidence that obesity and ESR in young men is a risk factor subsequent development of colon cancer.

Studies show that adult BMI is positively associated with colorectal cancer risk, with a metaanalysis reporting that each $5 \mathrm{~kg} / \mathrm{m}^{2}$ increase in adult body mass index is associated with a $25 \%$ increase in risk of colorectal cancer among men, an association that is significantly stronger than that observed among women [37]. Chronic inflammation in adulthood was also implicated in the etiology of CRC, with prospective studies observing significant associations between CRC and inflammatory biomarkers, such as C-reactive protein (CRP) and prostaglandin E2-metabolite (PGE-M).

A recent report involving a study of global cancer occurrences between 2002 and 2012 showed that 481,000 cancer cases ( $3.6 \%$ of all new cancers) in adults over 30 were attributed to a high body mass index (>25) [36]. In this analysis, about two-thirds of cancer attributable to a body mass index $>25$ consisted of colorectal cancer, uterine cancer and postmenopausal breast cancer. in this study region-specific estimates show that all three Asian regions and sub-Saharan Africa had the lowest frequencies of Body mass index -related cancers, ranging from $0.4 \%$ to $0.9 \%$ of total cancers (3.6\% to $6.0 \%$ of total high-Body mass index -related cancers) in men and $1.7 \%$ to 
$3.0 \%$ of total cancers (5.4\% to $8.3 \%$ of total high- Body mass index -related cancers) in women. For the remaining regions (Middle East and North Africa, Latin America and the Caribbean, Oceania, and all European regions), the Body mass index - related cancer frequencies ranged from $2.0 \%$ to $9.9 \%$ of total cancers ( $14.4 \%$ to $18.2 \%$ of high- Body mass index -related cancers) in both sexes [38].

According to Bhaskaran et al., high body mass index predisposes to several site-specific cancers, based on a study of 5.24 million individuals [39]. Of these, 166, 955 developed 22 different types of cancers of interest. Body mass index was associated with 17 of 22 cancer types, but effects varied substantially by primary cancer site. Increasing Body Mass Index was roughly linearly associated with cancers of the uterus (hazard ratio [HR] 1.62, 99\% CI 1.56-1.69; p<0.0001), gallbladder $(1.31,1.12-1.52 ; \mathrm{p}<0.0001)$, kidney $(1.25,1.17-1.33 ; \mathrm{p}<0.0001)$, cervix $(1.10,1.03-$ $1.17 ; \mathrm{p}=0.00035)$, thyroid $(1.09,1.00-1.19 ; \mathrm{p}=000088)$, and leukaemia $(1.09,1.05-1.13$; $\mathrm{p} \leq 0.0001)$.Body mass index was also positively associated also with liver $(1.19,1.12-1.27)$,

colon $(1.10,1.07-1.13)$, ovarian $(1.09,1.04-1.14)$, and postmenopausal breast cancers $(1.05$, 1.03-1.07) [all $\mathrm{p}<0.0001]$.

\section{Mechanisms Linking inflammatory disease and colorectal cancer}

The GI tract which is constantly exposed to various pathogens and food antigens can induce a series of host defensive reactions such as recruitment of immune cells, activation of antiinflammatory enzymes and secretion of anti-inflammatory cytokines. Fig.1 demonstrates that TGF $\beta$ has a role in the pathogenesis of IBD, and it also may contribute to the cancer risk associated with chronic inflammation of the gut. 
TGF $\beta$ in the presence of IL-6, which is produced during active infections or other inflammatory states, leads to the differentiation of naïve $\mathrm{T}$ cells into $\mathrm{Th}-17$ cells, a set of $\mathrm{T}$ helper cells secreting IL-17, a potent inflammatory cytokine. The production of IL-17 leads to chemokine expression such as IL- 6 and TNF $\alpha$. Th-17 appears to play an important role in IBD, and drives a procarcinogenic process under poorly regulated pro-inflammatory conditions (Fig.1) [40].

TGF $\beta$ also contributes to epithelial to mesenchymal transition (EMT), a hallmark that facilitates metastasis and invasion through multiple signaling mechanisms, leading eventually to tight junctions (TJ) and adherent junctions (AJ) dissolution. Another mechanism, by which TGF $\beta$ facilitates EMT, is through the up regulation of metallo proteinases (MMPs), contributing to colon carcinogenesis and metastasis (Fig.1).

In a study conducted by Hawinkels et al. in 2014, a strong activation of TGF- $\beta /$ Smad signaling pathway was observed in CRC. TGF- $\beta 1$ stimulation caused a remarkable up regulation of MMPs, plasminogen activatorinhibitor-1 (PAI-1) and TGF- $\beta 1$ itself, thus, leading to cumulative production of TGF- $\beta$ and proteinases with in the tumor microenvironment and consequently creating a cancer-promoting feedback loop (Fig.1).

There are several lines of evidence posing NFKB as a key regulator in the crosstalk among the pathways leading to T2DM, CRC, and IBD. Actually, various carcinogens, growth factors, inflammatory stimuli including microbiota and pro-oxidants activate the transcription factor NFkB which plays a central role in inflammation and is mostly expressed in cancers. It was documented that NFkB is activated via phosphorylation of inhibitor of kappa B (IKB) leading to its ubiquitination and proteasomal degradation. Such a reaction will unmask the nuclear localization signal of NF-KB, and once in the nucleus, it will activate several genes that regulate 
proliferation, apoptosis, angiogenesis, invasion, inflammation and metastasis [34], (Fig.2).NFKB plays a major role in linking chronic inflammation to cancer development through its ability to up regulate several inflammatory, and tumor promoting cytokines such as IL-6, IL-1 $\alpha$ and TNF$\alpha$, as well as survival genes such as BCL2 and BCLXL (Fig.2) [2,30] .

In addition, NFkB promotes EMT, through its activation of snail and twist in the microenvironment, and the expression of inflammatory cytokines. Moreover, NFKB seems to be involved in the tumor associated macrophage (TAM) recruitment and acts in cancer-associated fibroblasts (CAF), by promoting the expression of a pro-inflammatory gene signature, which is important for macrophage recruitment, neovascularization and tumor growth that were abolished when NFKB was inhibited (Fig.2).

Furthermore, NFKB plays an important role in T2DM, as obesity activates the transcription factor NFKB which increases the risk forT2DM. It has been shown that NFKB pathway inhibition exerts a beneficial effect on T2DM, salsalate a prodrug that reduces NFkB activity was shown to decrease HBA1C levels .Collectively, NFKB could be considered as the matchmaker between inflammation, IBD, cancer and diabetes, and its wide mechanisms of action are summarized in Fig.2 [14,30].

TNF $\alpha$ is a cytokine produced by activated macrophages as well as other cells. It is one of the first cytokines to be released during the acute inflammatory response. It plays a pivotal role in systemic inflammation as it induces the synthesis of C-reactive protein, vasodilatation, and vascular permeability and helps in the recruitment of inflammatory cells. In harmony with IL-17, TNF- $\alpha$ triggers the expression of neutrophil-attacking chemokines CXCL1, CXCL2 and CXCL5 
which in turn increase CXCR2-dependent neutrophil migration to the inflammation site. Diapedisis is also facilitated through TNF- $\alpha$ 's effect on cell adhesion molecules [31] (Fig. 3).

Among the immune-regulatory factors, TNF $\alpha$ is increased in the mucosa of IBD patients, and has been shown to play a central role in the pathogenesis of the disease, and anti TNF $\alpha$ therapy has proven to be beneficial in the treatment of IBD . In addition, TNF $\alpha$ activates other signaling pathways such as NFkB and Mitogen-activated protein kinases (MAPK) pathway, resulting in Jun N-terminal kinase (JNK) and the activator protein 1 (AP-1) activity. Thus the sustained activity of both NFKB and AP-1 is known to be a mediator in distinct phases of colon carcinogenesis (Fig.3) [31].

More than a decade ago, it was discovered that TNF $\alpha$ is over expressed in the adipose tissues of obese mice, thereby establishing a clear link between obesity, T2DM and chronic inflammation. Consequently, as represented in Fig.2, TNF $\alpha$ is a key player in IBD, CRC and T2DM.The association between T2DM and cancer is validated in several studies (35). (Hiroshi et al.,2012) demonstrated from a meta-analysis of 15 studies including more than 2.5 million patients that diabetic people have $30 \%$ increased risk of developing CRC compared to non-diabetics .371 million people worldwide have DM [40].

On the other hand several studies investigated the correlation between T2DM and IBD in 2015, SY LEE et al showed that metformin which is an effective widely used T2DM treatment was able to decrease the severity of IBD and diminishes inflammation [41]. 


\section{CVD Association and Metabolic Syndrome with Colorectal Cancer}

Several cohort studies have documented the adverse cardiovascular health effects experienced by overweight adults. In part, cardiac failure develops as a consequence of the increased demands on the heart to supply blood to the increased body fat; adipokines also increase the risk of cardiovascular complications of obesity [42]. The Nurses Cohort Study demonstrated a 2-fold increase in coronary heart disease in overweight women and a 3.6-fold increase with obesity. Similarly, the Framingham Heart Study showed increased incidence in heart disease proportionate to excess weight. Adult weight gain is an additional risk. For example, compared with men and women who maintained weight to within $2 \mathrm{~kg}$ of their weight at age $18-20$, those who gained 5-9.9 kg experienced a 1.5- to 2-fold higher risk of coronary heart disease and hypertension [43].

The fact that the metabolic syndrome is a risk factor for both CVD and colorectal cancer raised the question if there is any association between CVD and colorectal cancer. This correlation was found to be positive in several studies. In a pilot study of 63 patients with colorectal cancer were, demonstrated a statistical association between CVD and colorectal cancer in men [44]. Diabetes Association and the European Association for the Study of Diabetes for several reasons: Criteria are ambiguous or incomplete (for example it is unclear if the blood pressure definition is systolic blood pressure $\geq 130 \mathrm{mmHg}$ and diastolic $\geq 85 \mathrm{mmHg}$ or whether it is either $\geq 130 \mathrm{mmHg}$ or $>$ $85 \mathrm{mmHg}$ ); The value of including diabetes in the definition is questionable and the role of insulin resistance as unifying etiology is uncertain. Furthermore it is still unclear the extent to which an elevated cardiovascular (CVD) risk is due to insulin resistance itself vs isolated hyperinsulinemia [43]. 
Applying the ATP III criteria to 10537 NHANES III participants resulted in a significant association between the metabolic syndrome with prevalent myocardial infarction and stroke in a multivariate analysis: myocardial infarction [OR: 2.01, 95\% confidence intervals (CI): 1.532.64], stroke (OR: 2.16, 95\% CI: 1.48-3.16), and myocardial infarction/stroke (OR: 2.05, 95\% CI: 1.64-2.57) [9].

There is no clear basis for including/excluding other CVD risk factors; the CVD risk associated with the metabolic syndrome appears to be no greater than the sum of its parts; the treatment of the metabolic syndrome is not different from the treatment for each of its components[42]. The relationship between individual components of metabolic syndrome and the risk of colorectal cancer was also separately analyzed by several studies, which found a 35\% increased risk of colorectal cancer associated with high blood pressure. The results were confirmed by another study. Both studies also underlined that the clustering of metabolic syndrome components significantly increased the risk of associated colorectal cancer[45].

\section{Significance of the study}

There are limited studies conducted with regard to the prevalence of metabolic syndrome among colorectal carcinoma in Ethiopia. Therefore determining the prevalence of metabolic syndrome among colorectal carcinoma was a great benefit to study area.

The study was provide information on the associations between colorectal cancer and individual components of the metabolic syndrome; therefore, I was used these data to examine whether or not individual components of the metabolic syndrome altered colorectal cancer risk. Based on the finding, strategy for diagnosis, prevention and control method was suggested so as to prevent further complication and death due to colorectal cancer. 


\section{MATERIALS AND METHODS}

\section{Study area and period}

The study was conducted at adult oncology unit of TASH. TASH is a large referral teaching hospital, under the administration of Addis Ababa University, located in Addis Ababa, Ethiopia. TASH has divisions such as internal medicine, surgery, gynecology and obstetrics, pediatrics, radiotherapy, adult oncology, pediatric oncology /hematology, nuclear medicine, psychiatry, laboratory, orthopedics and pharmacy. The hospital has more than 800 beds, give diagnostic, and treatment service for about $370,000-400,000$ patients per year. The oncology unit of TASH is the only oncology unit for the country and has an outpatient department, which gives service to new and follow-up patients, and an in-patients department, which has 19 beds. The study was conducted in TASH from February, 2016 to June 2017 central Ethiopia, Addis Ababa.

\section{Study Design}

A cross sectional study with comparative nature was conducted on colorectal cancer individuals in Tikur Anbesa specialized hospital (TASH).

\section{Population}

\section{Source population}

The source population constitutes all colorectal cancer patients who attended oncology unit of Tikur Anbesa specialized hospital (TASH). 


\section{Study population}

The study population constitutes all colorectal cancer patients who attended the oncology unit of TASH from that fulfill the inclusion criteria of the study February, 2016 to June 2017 central Ethiopia.

\section{Inclusion and exclusion criteria}

\section{Inclusion}

All colorectal cancer patients who were volunteer to including in this project

All colorectal cancer patients who were not taken chemotherapy for diabetics, hypertension, cholesterol lowering and CVD

\section{Exclusion}

Patients who were diagnosed with other cancer

Colorectal cancer Patients who had had known CVD.

Hemolysed Blood sample

\section{Calculation of Statistically Recommended Number of Patients}

The sample size was calculated based on an estimated $5.7 \%$ prevalence of colorectal cancer in Ethiopia [46, 47]. The calculated sample size, using the following formula, was 83 colorectal cancer patients: In the calculation sample size, the following points are considered.

$\mathrm{P}=$ estimates of prevalence rate for the population $=5.7 \%$

$\mathrm{D}=$ the margin of sample error tolerated $=5 \%$

$\mathrm{Z} \alpha /_{2}$ is the standard normal variable at $1-\alpha \%$ confidence level $=1.96$ and $\alpha=5 \%$ 
$\mathrm{q}=1-\mathrm{p}$

$\mathrm{n}=$ minimum sample size $=$ ?

Based on the above assumption, the desired sample size is determined by the single population formula as follow;

$$
\begin{aligned}
\mathrm{n} & =(Z \alpha / 2)^{2} \mathrm{p}(1-\mathrm{p}) / \mathrm{d}^{2} \\
& =(1.96)^{2} 0.057(1-0.057) /(0.05)^{2} \\
\mathrm{n} & =83
\end{aligned}
$$

\section{Sampling technique}

The sampling technique was a conventional sampling technique in which all age group that has colorectal carcinoma available during the study periods was included.

\section{Variables}

\section{Dependent variable}

- Colonoscopy analysis result.

\section{Independent variable}

Age, Sex, obesity, Family history, Fasting glucose test, Blood pressure, Smoking habits, eating habits and metabolic syndrome

\section{Procedure for data collection}

The data was collected by trained nurses with using well-structured questionnaire which contain the required information; there for the sours of data was both questionnaire and Laboratory investigation. 


\section{Data processing and analysis}

The value of different variables was compiled on a tally sheet and using tables the result was calculated by statistical software for social science (SPSS) V 21, the association between metabolic syndrome and cardiovascular risk factor association with colorectal cancer was compared with ATP III and Framingham risk score.

\section{Sample collection and handling}

Information about the study was explained to the patients and enough blood samples was drown in to chemistry analyzing test tube for chemistry and Blood glucose analyses.

A standard mercury sphygmomanometer was used to measure blood pressure after participants was allowed to sit at least $5 \mathrm{~min}$. then about $5 \mathrm{ml}$ blood was drown, by vain puncture from the medial cubital vein, after an overnight fasting. The entire blood sample was taken by a trained health professional using universal precautions. Blood samples was left to clot for 1 to $2 \mathrm{hrs}$ then samples centrifuged for $10 \mathrm{~min}$ at $3000 \mathrm{rpm}$, and the supernatant (serum) was removed and stored at frozen at $-70^{\circ} \mathrm{C}$ until analysis.

\section{Anthropomorphic Measurements and Biochemical Assays}

\section{Anthropometric measurements}

Waist circumference was measured using a measuring tape at the level of the superior part of the umbilicus. Body mass index (BMI) was calculated as weight in kilograms divided by the squire of height in meters and categorized as underweight $\left(<18 \mathrm{~kg} / \mathrm{m}^{2}\right)$, normal $\left(18-25 \mathrm{~kg} / \mathrm{m}^{2}\right)$, over weight $\left(25.0-29.9 \mathrm{~kg} / \mathrm{m}^{2}\right)$, and obese $\left(\geq 30.0 \mathrm{~kg} / \mathrm{m}^{2}\right)$. 


\section{Methods of Biochemical assays}

Serum samples were analyzed for fasting plasma glucose, triglycerides, total cholesterol, HDL cholesterol and LDL cholesterol in the biochemistry laboratory of Tropical research center, Assela. The reagents were commercially available in readymade kits.

\section{Determination of Fasting Serum Glucose}

Fasting serum glucose levels was determined by enzymatic glucose oxidase method using commercial reagents kit

Principle: In the Trinder reaction the glucose is oxidized to D-gluconate by the glucose oxidase (GOD) with the formation of hydrogen peroxide. A colorless mixture of phenol and 4 aminoantpyrine(4-AA) is then oxidized by hydrogen peroxide in the presence of the enzyme, peroxidase, to form a red quinoneimine dye product. The concentration of the coloured product, determined from the optical density change at $540 \mathrm{~nm}$ in the reaction, is proportional to the concentration of glucose in the original serum sample.

B-D - Glucose $+\mathrm{H} 2 \mathrm{O} \stackrel{\text { GOD }}{\longrightarrow}$ D - Gluconate $+\mathrm{H} 2 \mathrm{O} 2$

$4-\mathrm{AA}+$ phenol $\underset{\text { POD }}{\stackrel{\mathrm{H} 2 \mathrm{O} 2}{\longrightarrow}}$ Quinoneimine dye $+\mathrm{H} 2 \mathrm{O}$

\section{Reagent composition}

R1 Monoreagent. Phosphate buffer $100 \mathrm{mmol} / \mathrm{L} \mathrm{pH} \mathrm{7.5,} \mathrm{glucose} \mathrm{oxidase} \mathrm{>} \mathrm{10KU/L,} \mathrm{peroxidase}$ $>2 \mathrm{KU} / \mathrm{L}, 4$-aminoantipyrine $0.5 \mathrm{mmol} / \mathrm{L}$.

Glucose standard: Glucose $100 \mathrm{mg} / \mathrm{dl}$ organic mixture based primary standard. 
Procedure: Ten microliters of serum was mixed in a cuvette with $1 \mathrm{ml}$ of glucose reagent $\mathrm{R} 1$, and then incubated at room temperature for 10 minutes. Then the optical density was read at 540nm, against a reagent blank. Standard glucose concentrations were used to determine the specific concentration of glucose in serum samples, according to the Lambert-Beer law.

\section{Determination of Serum Triglyceride Level}

Principle: The method is based on the enzymatic hydrolysis of serum or plasma triglyceride to glycerol and free fatty acids (FFA) by lipoprotein lipase (LPL). The glycerol is phosphorylated by adinosine triphosphate (ATP) in the presence of glycerol kinase (GK) to form glycerol-3phosphate (G-3-P) and adenosine diphosphate (ADP). G-3-P is then oxidized by glycerol phosphate oxidase (GPO) to form dihyroxyacetone phosphate (DHAP) and hydrogen peroxide. A red coloured product is formed by the peroxidase (POD) catalyzed coupling of 4aminoantipyrine (4-AA) and phenol with hydrogen peroxide (H2O2), the optical density at 540 $\mathrm{nm}$ of which is proportional to the concentration of triglyceride in the sample.

Triglycerides $+3 \mathrm{H} 2 \mathrm{O} \stackrel{\text { LPL }}{\longrightarrow}$ Glycerol $+3 \mathrm{FFA}$

Glycerol + ATP $\longrightarrow$ GK $\longrightarrow$ Glycerol -3-P + ADP

Glycerol -3-P $+\mathrm{O}_{2} \stackrel{\text { GPO }}{\longrightarrow}$ DHAP $+\mathrm{H}_{2} \mathrm{O}_{2}$

4- $\mathrm{AA}+4 \mathrm{Phenol} \stackrel{\mathrm{H} 2 \mathrm{O} 2}{\longrightarrow}$ Quinoneimine $+\mathrm{H}_{2} \mathrm{O}$

\section{Reagent composition}

$\mathbf{R} 1=$ Mono reagent pipes buffer $50 \mathrm{mmol} / \mathrm{L} \mathrm{pH} 6.8, \mathrm{LPL} \geq 12 \mathrm{KU} / \mathrm{L}, \mathrm{GK} \geq 1 \mathrm{KU} / \mathrm{L}, \mathrm{POD} \geq$ 2.5KU/L4-AA 0.5mmol/L phenol $3 \mathrm{mmol} / \mathrm{L}$, non - ionictensioactives $2 \mathrm{~g} / \mathrm{L}$ Biocides. 
Triglycerides standard: - Glycerol $2.25 \mathrm{mmol} / \mathrm{L}$, equivalent to $200 \mathrm{mg} / \mathrm{dl}$ of glycerol trioleate,

Procedure: -Ten micro liter of serum was mixed in a cuvette with $1 \mathrm{ml}$ of triglyceride mono reagent $\mathrm{R} 1$, and then incubated at room temperature for 10 minutes. Then the optical density was read at 540nm, against reagent blank and compared with standard triglyceride concentrations used as samples.

\section{Serum Total Cholesterol Determination}

Principle: The method for the measurement of serum total cholesterol involves the use of three enzymes: cholesterol esterase (CE), cholesterol oxidase (CHOD) and peroxidase (POD). Cholesterol esters are first hydrolyzed to release free cholesterol and triglycerides using cholesterol esterase. The free cholesterol is then oxidized by CHOD to generate H2O2. The hydrogen peroxide reacts with phenol and 4-aminoantipyrine in the presence of peroxidase to generate a coloured quinoid dye product, the absorbance of which is measured at $540 \mathrm{~nm}$, and is

proportional to the concentration of total cholesterol in the original sample: Reagents: R1: 200 $\mathrm{mmol} / \mathrm{L}$ PIPES $\mathrm{pH} 7.0$, containing $1 \mathrm{mmol} / \mathrm{L}$ sodium cholate, > $250 \mathrm{U} / \mathrm{L}$ cholesterol esterase, $>250 \mathrm{U} / \mathrm{L}$ cholesterol oxidase, $>1 \mathrm{KU} / \mathrm{L}$ peroxidase, $0.33 \mathrm{mmol} / \mathrm{L}$ 4-aminoantipyrine, $4 \mathrm{mmol} / \mathrm{L}$ phenol, $2 \mathrm{~g} / \mathrm{L}$ non-ionic surfactant, and commercial biocides.

$\mathrm{R} 2: 5.18 \mathrm{mmol} / \mathrm{L}$ cholesterol standard.

Procedure: $1.0 \mathrm{ml}$ of the working reagent (R1) was mixed with $10 \mu \mathrm{l}$ of serum sample or $10 \mu \mathrm{l}$ of standard cholesterol solution. After 5 minutes of incubation at $37^{\circ} \mathrm{C}$, the absorbance was measured at $540 \mathrm{~nm}$ against the reagent blank.

Serum Total Cholesterol Concentration Calculation: Serum total cholesterol concentration was calculated as follows: 
Con. Total Cholesterol $(\mathrm{mg} / \mathrm{dL})=($ A sample $/$ A standard $) X$ Concentration of standard

Where $\mathrm{A}$ is the absorbance at $546 \mathrm{~nm}$ of solutions after reactions were completed.

\section{HDL-Cholesterol}

Principle: - This technique a separation method based on the selective precipitation of Apo lipoprotein B-containing lipoproteins (mainly VLDL and LDL) by phospotungstic acid/ $\mathrm{MgCl}_{2}$, Sedimentation of the preciptate by centrifugation, and subsequent enzymatic analysis of high density lipoprotein (HDL) as residual cholesterol remaining in clear supernatant.

\section{$\underline{\text { Reagent composition }}$}

Precipitating Reagent, Phosphotungstic acid $0.63 \mathrm{mmol} / \mathrm{L}$ magnesium chloride $25 \mathrm{mmol} / \mathrm{L}$ Stabilizers. Cholesterol standard, $50 \mathrm{mg} / \mathrm{dL}$

\section{Procedure}

\section{I, Precipitation}

1. $0.2 \mathrm{ml}$ of serum was mixed with $0.4 \mathrm{ml}$ of Precipitating Reagent in a test tube then allowed to stand for $10 \mathrm{~min}$ at room temperature. The sample was then centrifuged for 10 min. at 10,000 rpm and the supernatant containing HDL removed and further processed for cholesterol determination.

\section{$\underline{\text { II Colorimetry }}$}

50 microliter of supernatant containing HDL was mixed in a cuvette with $1 \mathrm{ml}$ of cholesterol MR mono reagent $\mathrm{R} 1$, and then incubated at room temperature for 10 minutes. Then the optical 
density was read at $540 \mathrm{~nm}$, against reagent blank, as described under the method for total cholesterol determination.

\section{LDL - CHOLESTEROL}

A method developed by Friedwald's was used to estimate LDL-C level in The method involves measurements of fasting total cholesterol, triglyceride, and high-density lipoprotein cholesterol concentrations and calculating the value of LDL-c by using Friedwald's formula. It may not reliable at very low LDL cholesterol concentrations and /or very high ( $>400 \mathrm{mg} / \mathrm{dl})$ triglyceride values.

In Friedwald's formula, TG replaces VLDL, because it has been shown that serum TG levels are equivalent to five times the levels of VLDL.

LDL cholesterol was determined using the Friedewald formula

Friedwald's formula: LDL-C = Total Cholesterol - [HDL-C + (TG/5)]

\section{Smoking and Framingham Risk Scores}

Of the 79 colorectal cancer patients, 8 were cigarette smokers. Colorectal cancer patients who smoke are at a significantly increased risk of cardiovascular disease compared with colorectal cancer patient non-smokers. The Framingham risk score uses the following parameters: sex (male or female), age, smoking status (yes or no), systolic blood pressure (in $\mathrm{mm} \mathrm{Hg}$ ), use of blood pressure medications (yes or no) were calculated for smokers only, serum triglyceride (in $\mathrm{mg} / \mathrm{dL}$ ) and serum total cholesterol (in $\mathrm{mg} / \mathrm{dL}$ ) to calculate the percentage risk of a person suffering from a myocardial infarction during the next ten years. It is based on a study of tens of thousands of individuals over many decades in the USA, but can be generally applied to other populations [48]. 
The risk was based on a complex equation, and can be determined by inserting the patient's parameters into an online site; the risk factor was then determined automatically.

\section{RESULTS}

Demographics of colorectal cancer patients in the study

The study was done on colorectal cancer patients (no control non-cancer patients) attending the Oncology Clinic at Tikur Anbessa Specialized Hospital. The goal was to assess the prevalence in these colorectal cancer patients of cardiovascular risk factors, other parameters relating to metabolic syndrome, and Body Mass Index (BMI).

A total of 79 patients were included in the study. More than one third 25 (31.6\%) of the patients were in the age range of 40-49 years, with a mean age of 46.9 years and more than a half $41(51.9$ \%) were from Addis Ababa as shown in Table 2.

\section{Clinical characteristics of the study subjects}

Table 4 shows that, of the 79 colorectal cancer patients, colon cancer occurred in 42 (53.2\%) and rectal cancer occurred in 37 (46.8\%) of patients. Colon cancer was more prevalent in males than rectal cancer, and the opposite was true for females, where rectal cancer was more prevalent than colon cancer.

TNM staging, which is based on local tissue tumor $(\mathrm{T})$, lymph node metastases $(\mathrm{N})$ and distant metastases (M), of the colorectal cancers in different patients in the study indicated that all stages from I to IV were represented (Table 5). See Introduction (Table 1) for definitions of staging. 
Thus, 10/79 (12.7\%) of patients had Stage I; 32/79 (40.5\%) had Stage II disease (IIa, IIb or IIc); 20/79 (25.3\%) patients had Stage III (IIIa, IIIb, IIIc) disease; and 17/29 (21.5\%) had Stage IV disease. In other words, most patients $(87.3 \%)$ had cancer at Stage II or beyond.

Table 6. Shows the stage of colorectal cancer categories in patients with either colon or rectal cancer. The results show that every stage (I to IV) is represented in both colon and rectal cancer patients. In addition, 18 (48.6\%) of rectal cancer patients presented with Stage I or II disease, whereas $24(57.1 \%)$ of colon cancer patients presented with Stage I or II disease. The numbers of patients in each stage were relatively low, so statistical analysis was not informative.

\section{Results of measure BMI and metabolic syndrome components}

Table 7. Shows the body mass index (BMI) for the colorectal cancer patients studied. For males, 21/46 (45.7\%) were either overweight (41.3\%), with a BMI between 25 and 29.9 or obese $(4.3$ $\%$ ), with a BMI of 30 or greater. For females, 14/33 (42.4\%) were either overweight (39.4\%) or obese $(3.0 \%)$. For males and females combined, $44.3 \%$ were overweight or obese.

The numbers and percentages of patients who had abnormal parameters of metabolic syndrome showed by table- 8 .

Considering the diagnosis of metabolic syndrome as the presence of three or more of the following: (1) elevated waist circumference (Male > $102 \mathrm{~cm}$, Female > $88 \mathrm{~cm}$ ); (2) hypertriglyceridemia ( $>150 \mathrm{mg} / \mathrm{dl}$ ); (3) low HDL-C (male $<40 \mathrm{mg} / \mathrm{dL}$, female $<50 \mathrm{mg} / \mathrm{dL}$ ); (4) elevated blood pressure (>130 $\mathrm{mm} \mathrm{Hg}$ systolic or $\geq 85 \mathrm{mmHg}$ diastolic), and (5) increased blood 
glucose $(>100 \mathrm{mg} / \mathrm{dl})$, the numbers and percentages of the colorectal cancer patients with metabolic syndrome are also indicated in Table 8 .

The data shows that almost one third 23 (29\%) of colorectal cancer patients have metabolic syndrome by these criteria. Almost a half (45.6\%) of patients had an elevated blood pressure (either systolic or diastolic or both), whilst 53/79 (67.1\%) of all patients had a fasting blood glucose over $100 \mathrm{mg} / \mathrm{dL}$, which is an abnormal component of the metabolic syndrome. Of the 53 patients with blood glucose over $100 \mathrm{mg} / \mathrm{dL}, 31(39.2 \%)$ patients had serum fasting blood glucose levels greater than $126 \mathrm{~g} / \mathrm{dL}$, which is a cut-off value for diagnosing diabetes used by many medical authorities. Approximately two-thirds (63.3\%) of patients had decreased serum HDL levels, and more than half (57\%) of patients had elevated serum TG level.

\section{Framingham scores and smoking status of colorectal cancer patients}

The Framingham Risk Score is a calculated number based on empirical data, from which an equation has been derived that predicts the percentage chance of a person having their first myocardial infarction in the next ten years. The score is based on a person's age, sex, serum total cholesterol, serum HDL, blood pressure, and whether they are a smoker or not. (48).

Framingham scores were calculated for all colorectal cancer patients where the equation applies. Though the standard deviations were high, Framingham scores, as expected, were lower for nonsmokers than for smokers (Table 9).

Smokers' Framingham risk scores are shown in Table 10, and the range is wide, but four smokers had a very high risk (over $15 \%$ chance) of having a heart attack in the next ten years. If these patients were to quit smoking, their risks would fall substantially, though it can take months to a few years for risks to fall to minimum values. 


\section{Levels of LDL cholesterol and other cardiovascular risk factors}

Serum LDL is also a risk factor for cardiovascular disease. Generally, a serum LDL greater than $130 \mathrm{mg} / \mathrm{dL}$ has been considered a risk in the general population, although studies with diabetics suggest that the goal should be less than $100 \mathrm{mg} / \mathrm{dL}$, and with individuals with known cardiovascular disease LDL should be below $70 \mathrm{mg} / \mathrm{dL}$.

Taking a serum LDL of $>130 \mathrm{mg} / \mathrm{dL}$ as a significant risk for cardiovascular disease, it was found that $18 / 46(39.9 \%)$ of male colorectal cancer patients, and 12/33 (36.4\%) females in this study had an LDL $\geq 130 \mathrm{mg} / \mathrm{dL}$ (Table 11$)$.

Other results for cardiovascular risk factors in these colorectal cancer patients showed that 37/79 $(46.8 \%)$ of patients ate red meat regularly; only one patient reported a family history of cardiovascular disease; and 59/79 did less than twice a week of reasonable exercise.

\section{DISCUSSION}

In Ethiopia, cancer accounts for about $5.8 \%$ of total national mortality. Although populationbased data do not exist in the country except for Addis Ababa, it is estimated that the annual incidence of cancer is around 60,960 cases and the annual mortality is over 44,000 [49]. Cost effectiveness analyses of the many interventions (primary prevention, screening or treatment) for reducing the burden of colorectal cancer have usually been restricted to developed country settings and with often considerable variation in the analytical methods used. This limits the value of the existing literature to inform colorectal cancer control policies in low to middle income country like Ethiopian settings. 
The mean age of the colorectal patients in this study was $46.9 \pm 14.8$ years. The age range was from 20 to 78 years for both males and females; $64.6 \%$ of the patients were below the age of 50 years. Peak age incidence of colorectal cancer in this study was 38 to 45 which is much lower than that of other countries. For example, in Bangladesh $58 \%$ of colorectal cancer patients were below the age of 50 years (mean age $47 \pm 14.8$ years) [50]. In a study from Jamaica, $20 \%$ of the overall group was less than age 50 [51]. Turner et al., 2010, found only $20 \%$ of the cases below 50 years in USA. The mean age of colorectal cancer patients in this thesis study suggests that colorectal carcinoma is relatively common in a lower age group in Ethiopia. This may be due to both environmental factors, such as carcinogens chemicals in food and water (exposed to aflatoxin and high chlorinated water) and genetic factors.

The finding that $64.6 \%$ of the overall group was less than age 50 years is important because most medical organizations like CDC and WHO recommend screening colonoscopies and other screening protocols to start at age 50 in the general population [52-53], and this may have to be revised in Ethiopians to start screening earlier than 50 years old. Otherwise, patients under 50 years would be more likely to present with symptomatic disease at a more advanced stage, and with a poor prognosis.

One possible environmental cause of colorectal cancer is long-term (over 35 years) exposure to heavily chlorinated drinking water, which contains carcinogenic trihalomethanes. Tri halo methanes concentrations at a level of approximate $75 \mathrm{mg} / \mathrm{L}$ or higher were associated with a double colon cancer risk among men. The highest quartile of cumulative trihalomethanes-years exposure was associated with an OR of 1.7 [54-55]. 
During the chlorination process, chlorine reacts with organic materials in the water to produce a complex mixture of halogenated and non-halogenated by-products, the concentration and distribution of which vary with characteristics of the raw water and the treatment process. A large number of halogenated chemical species have been identified, including tri-halo methanes, halogenated acetonitriles, halogenated acids, halo ketones, and halo aldehydes [56]. Trihalomethanes are the most frequently occurring by-products and are routinely measured in public water supplies, thus making them a useful marker for the level of chlorination by-products in treated water. Chronic exposure to disinfected surface water is considered one of the contributing factors to the development of urinary bladder and colon cancer [54-56].

Another possibility is that exposure to aflatoxins might contribute to colon cancer. Aflatoxins are known to be present in various Ethiopian cereal crops and other agricultural products (for example, peanuts and tobacco), and aflatoxins have also been found in milk in Ethiopia. Although there is little evidence that aflatoxins (which cause mainly liver cancer) cause colon cancer, some studies in rats suggest that a vitamin A-deficient diet in rats exposed to aflatoxin B1 (AFB1) was causally related to colon cancer [57-58]. Whether or not this is true for humans is not known, but it does raise the possibility that aflatoxin exposure in Ethiopia might, under some circumstances, increase the risk of colorectal cancer.

Some studies showed that black men had a higher colorectal cancer prevalence compared with white men at 50-54 years $(\mathrm{OR}=1.17), 55-59$ years $(\mathrm{OR}=1.16)$, and 60-64 years $(\mathrm{OR}=1.38)$. There were similar findings for black women, who had a higher prevalence of large polyps at age 50-54 years, 55-59 years, and 60-64 years [59].

In the present study $89.9 \%$ of study participants come from urban area of Addis Ababa, Oromiya and Amahra. More than half of patients (51.9\%) come from Addis Ababa. this figure 
shows that urban life may contribute to colorectal cancer by more than one factors such as exposure to environmental pollution, aflatoxins and Trihalomethanes.

Recent meta-analysis studies done in Australia urban cities of total trihalomethane exposure and colon and rectal cancers [54] found that risks of both were increased in the highest category of exposure relative to the lowest: colon cancer OR $1.33(95 \%$ CI 1.12, 1.57) and rectal cancer OR $1.30(95 \%$ CI $1.06,1.59)[55-56]$.

Ethiopia is also affected by environmental pollution, particularly in big cities such as Addis Ababa, but also there is exposure of people in rural areas, where charcoal and wood are commonly used in and near homes, and people are exposed frequently to the smoke from the burning of these fuels for cooking and other purposes. In addition, indoor cigarette smoking may contribute to carcinogen exposure in rural areas.

A study also supported by the International Agency for Research on Cancer has classified outdoor air pollution as a carcinogen, conclusively linking it to lung and bladder cancers. A few studies have also strongly suggested a possible link between colorectal cancer and chemical and pesticide pollution of food. The Agricultural Health Study from the United States has demonstrated an elevated risk of colorectal cancer with exposure to pesticides. Dumping sites for industrial wastes are largely unregulated in most low-income countries like Ethiopia and can also potentially result in soil and water pollution, with consequent health hazards. One study found that concentrations of polychlorinated di benzo-p-dioxins, polychlorinated dibenzofurans, and coplanar polychlorinated biphenyls in soils from dumping sites in the Philippines, Cambodia, India, and Vietnam often exceeded environmental guideline values [60]. 
In this study, stage of cancer (stage II and higher) accounted for a relatively high percentage of patients (about 87\%). In patients with colon cancer 91\% were in an advanced stage (Stage II or higher), whereas $83.7 \%$ of rectal cancer patients had Stage II or higher. This difference might be due especially to the fact that polyps formed in ascending colon are painless and do not cause constipation because stool is liquid in this part of the colon, so right-sided colon cancer is often asymptomatic until it is more advanced in stage. Right colon cancer tends to present more with anemia as the tumours grow large and bleed. Left sided colorectal cancer tends to present earlier, with pain, constipation and bright blood in the stool.

In study done in Brazil, colorectal cancer was located in the colon in $56.7 \%$ of the cases, followed by rectum (36.2\%) and rectosigmoid junction (7.1\%). Most cancers $84.2 \%$ ) were at Stage II or higher, in general agreement with the results of this thesis [61].

Another study done in Scotland $25.1 \%$ of people were diagnosed with colorectal cancer at the earliest stage (stage I), and three-quarters were diagnosed at Stage II or higher. The most common stage of disease at diagnosis for colorectal cancer in Scotland generally was Stage II, which accounted for $26.4 \%$ of all patients. However, patients in the most socioeconomically deprived areas of Scotland had the highest proportion advanced stage of disease, stage IV $(29.6 \%)$.

In this thesis study a large number of patients were diagnosed at stage II (40.5\%) when compared with the Brazil (31.7\%) and Scottish (26.4\%) studies. The difference in this study might be due to lack of awareness of patients as to the seriousness of their symptoms or to lack of early screening, which is rare in Ethiopia due to poor availability of screening technology, only one oncology hospitals in the country and few oncologists. Early detection comprises early 
diagnosis of cancer in symptomatic populations and screening in asymptomatic high-risk and vulnerable populations. It is an approach that promotes vigilance for early signs and symptoms of disease. Early detection and treatment of cancer is known to reduce greatly the burden of colorectal cancers. Regular colorectal cancer screening is one of the most powerful ways to prevent colorectal cancer.

Chyke et al Also shows that the benefit of screening by compared with no endoscopic screening, screening colonoscopy was associated with a $67 \%$ reduction in the risk of death from colorectal cancer. By cancer location, screening colonoscopy was associated with a $65 \%$ reduction in risk of death for right-colon cancers and a $75 \%$ reduction for left-colon/rectal cancers[62].

In this study body mass index (BMI) data showed that $15 \%$ of patients were underweight, 40.5 $\%$ had normal BMI, $40.5 \%$ were overweight and $4.3 \%$ were obese. In the study of Daniel et al $54 \%$ of US colorectal cancer patients were obese [29], higher than that found in this thesis study, as expected because of the higher prevalence obesity in the US population [9]. Obesity was not therefore a major problem in the Ethiopian colorectal cancer population, but nevertheless the $40.5 \%$ of patients that were overweight are at significant risk of developing obesity, metabolic syndrome, cardiovascular disease and diabetes.

A WHO meta-analysis provided convincing evidence adult (Body mass index) BMI is positively associated with colorectal cancer (CRC) risk, the study reporting that each $5 \mathrm{~kg} / \mathrm{m}^{2}$ increase in adult $\mathrm{BMI}$ is associated with a $25 \%$ increase in risk of CRC among men, an association that is significantly stronger than that observed among women. Despite a large body of evidence linking adult $\mathrm{BMI}$ to $\mathrm{CRC}$ risk, comparatively few studies have evaluated the association between adolescent/young adult BMI and CRC. While the literature suggests a positive association, these 
studies largely relied on participant recall of earlier-life BMI or have been small and unable to disaggregate overweight and obese adolescents. There are a number of putative mechanisms by which BMI may affect the risk of CRC, including effects on insulin, leptin, steroid hormones and inflammation [38].

Metabolic syndrome is becoming increasingly common worldwide because of the epidemic of obesity and sedentary lifestyles. The biological plausibility of the association between metabolic syndrome and CRC may be mediated by dysregulation of growth signals [including insulin, insulin growth factor I (IGF-I), downstream signaling pathways, and adipokines], cytokines, and vascular integrity factors, thereby contributing to cancer-related processes [29].

Riondino et al described the role of hyperinsulinemia, IGF-I, and hyperleptinemia in the association between adiposity and CRC [A common pathway has also been suggested, in which these factors increase PI3K/Akt activity, which in turn regulates downstream targets, leading to reduced apoptosis, increased cell proliferation and survival, and promotes the cell cycle [64] . In addition, a recent paper reports that, among adipocyte-secreted hormones, the most relevant to colorectal tumorigenesis are adiponectin, leptin, resistin, and ghrelin. All these molecules have been involved in cell growth, proliferation, and tumor angiogenesis, and their expression changes from normal colonic mucosa to adenoma and adenocarcinoma [14].

In this study, $29 \%$ (12.7\% male, $16.5 \%$ female) of colorectal patients had metabolic syndrome (had more than 3 criteria of metabolic syndrome). Of all of the colorectal cancer patients, $45.6 \%$ had elevated blood pressure, which is concerning. This should be confirmed with repeated blood pressure measurement and if confirmed, patients should be treated with antihypertensive to lower the blood pressure. Of the colorectal cancer patients, $53(67.1 \%)$ had increase serum glucose level above $100 \mathrm{mg} / \mathrm{dL}$, which is concerning for diabetes. Of the 79 patients studied, a significant 
number $31(39.2 \%)$ of patients had a blood glucose above $126 \mathrm{mg} / \mathrm{dL}$, which defines diabetes according to some authorities. Therefore, all patients with elevated blood glucose should be retested for fasting blood glucose and those with diabetes should be treated with medications, diet and exercise regimens. Those with prediabetic blood glucose (100 to $126 \mathrm{mg} / \mathrm{dL}$ ) should be advised to control their diet and lifestyle to prevent them from becoming diabetics.

The patients who had low HDL cholesterol levels should be advised perform exercise regularly, and treated with a low fat, low cholesterol diet and if necessary treated with statins, which to some extent increase HDL. Those with elevated triglyceride levels should be advised to reduce their calorie and saturated fat intake and exercise more and consume foods rich in omega-3 fatty acids (especially fish). Those with elevated LDL levels should be considered for statin therapy, which substantially lowers LDL and decreases cardiovascular risk.

Daniel et al reported dose-response relationship between BMI and CRC; a 5-unit increase in BMI was related to an increased risk of colon cancer in both men and women. In addition, obesity has been more closely associated with CRC in men than in women. In a14-year multicentric cohort study, the authors reported a positive association between MS and CRC in men, but not in women [29].

Framingham Risk Score give an indication of the likely benefits of prevention, they are useful for both the individual patient and for the clinician in helping decide whether lifestyle modification and preventive medical treatment, and for patient education, by identifying men and women at increased risk for future cardiovascular events. Coronary heart disease (CHD) risk at 10 years in percent can be calculated with the help of the Framingham Risk Score. Individuals 
with low risk have $10 \%$ or less CHD risk at 10 years, with intermediate risk 10-20\%, and with high risk $20 \%$ or more [48].

The result of the present study clearly demonstrates that mean Framingham risk score of nonsmoker participants was very low risk (mean score $=8.2 \pm 6.6$ ) than smoker participants (mean score $=14.8 \pm 11.4$ ).If the smoker participants quit smoking, the mean risk was significantly reduced nearly by half (mean of smoker $=14.8$ reduced to mean $=8.2 \pm 6.6$ if quite). This emphasizes that smoking cessation is an important consideration in managing colorectal cancer patients, in order to reduce their cardiovascular risk.

It is stated that colorectal cancer risk is increased both in men and women with type 2diabetes and this is associated with colon cell proliferation triggered by hyperinsulinemia and increase free IGF- 1 levels. It is also shown that chronic insulin treatment is associated with increased colorectal adenoma risk in type 2 diabetic patients [64].

Physical inactivity may be responsible for $13-14 \%$ of colon cancer, an attributable risk greater than family history. Epidemiological evidence shows an association between occupational and recreational physical activity and colon cancer, but has not established whether physical activity is protective against low-risk or more advanced adenomas. The evidence is inconclusive as to whether physical activity protects against rectal cancer and is conflicting with respect to whether physical activity has equal effects on male and female risk of colorectal cancer. The effect of exercise 'interventions' on the risk of colorectal cancer is currently not known [65].

In this study exercise was done twice per week in only a quarter of patients. There is some evidence that improvements in cardiorespiratory fitness reduce adverse effects from cancer treatment when physical activity is undertaken following diagnosis of colorectal cancer. 
Markers/mechanisms by which physical activity may protect against colorectal cancer and/or improved disease prognosis include gastrointestinal transit-time, chronic inflammation, immune function, insulin levels, myokines, insulin-like growth factors, genetics and obesity.

In this study red meat eaten regularly by about a half of patients. Red meat has been linked to colorectal cancer \& cardiovascular disease so patients could be advised to eat red meat in moderation. Evidence also supports the idea that a diet that's high in red meat (beef, pork, lamb, or liver) and processed meats can raise colorectal cancer risk[66].

Red meat consists of compounds such as haem iron (HI) that facilitates the endogenous formation of N-nitroso compounds (NOCs) such as nitrosylated haeme iron, catalysing its formation from natural precursors in the gastrointestinal tract (GI) as well as through lipid peroxidation in the GI. In addition, Heam Iron (HI) can induce oxidative stress, colonocyte proliferation through the lipid-peroxidation pathway and induce the production of genotoxic free radicals in the colonic stream [67].

The carcinogenic compounds that form during processing and cooking include N-nitroso compounds (NOCs) and polycyclic aromatic hydrocarbons (PAHs). N-nitroso compounds (NOCs) are introduced exogenously from nitrates and nitrites added during the preservation process but can also be formed endogenously as alluded to previously. In processed meat, Heam Iron (HI) is nitroslated because curing salt contains nitrate or nitrite. There is evidence that nitrosylated Heam Iron (HI) promotes carcinogenesis at doses that are five to six times lower than non nitrosylated HI [68-69]. 


\section{CONCLUSION}

The higher colorectal cancer incidence in the oncology unit of Tikur Anbessa Specialized Hospital was the younger subgroups of populations which suggests that a recent change in the environmental and other risk factors, that affected individuals who shared these exposures during life time. Therefore, Primary prevention is thus considered the most cost-effective way of combating colorectal cancer. Early detection and treatment of colorectal cancer is known to greatly reduce the burden of the disease not only improve outcomes enhancing early detection and the treatment of early-stage disease, but also streamlining referral of diagnosed cancer patients for better treatment.

Apart from diagnoses of colorectal cancer, even though metabolic syndrome was lower percentage based on ATP III in the study area; the fact that individual metabolic syndrome components such as increased fasting glucose, LDL, TG and decreased HDL might play pathogenic role such as cardiovascular complication, T2 diabetic mellitus and colorectal cancer.

\section{List of abbreviations}

CRC Colorectal cancer

BMI body mass index

ESR Erythrocytes sedimentation rate

WHO world health organization

FAP familial adenomatous polyposis

HNPCC hereditary non polyposis colorectal cancer

IGF.1 Insulin growth factor 


$\begin{array}{ll}\text { ATP-III } & \text { Adult treatment panel III } \\ \text { HDL } & \text { High density lipoprotein } \\ \text { MetS } & \text { metabolic syndrome } \\ \text { VEGF } & \text { vascular endothelial growth factor } \\ \text { IL6 } & \text { Interleukin } 6 \\ \text { TNM } & \text { Tumor Size, Nodal Involvement and Metastasis } \\ \text { GH } & \text { Growth hormone } \\ \text { NF-kB } & \text { nuclear factor kB } \\ \text { IBD } & \text { Inflammatory bowel disease } \\ \text { T2DM } & \text { Type two diabetes mellitus } \\ \text { TAM } & \text { tumor associated macrophage } \\ \text { EMT } & \text { epithelial to mesenchymal transition }\end{array}$

\section{Declarations}

\section{Ethics approval and consent to participate}

Ethical consideration was approved by Department of Biochemistry Research And Ethical Review Committee meeting number DRERC: 06/15 with protocol number of M.Sc. thesis 2/15 in accordance with permission was obtained from oncology unit of TASH before start of data collection. The purpose and benefits of the study was explained to each study subject to come to consensus and those who have willing were included. 


\section{Consent for publication}

Not applicable

\section{Availability of data and materials}

The data sets used and /or analyzed during the current study are available from the corresponding authors or on reasonable request.

\section{Competing interests}

The authors declare that there is no conflict of interests.

\section{Funding}

The source of this research is Addis Ababa University. The fund is used data collection and diagnosis only.

\section{Authors' contributions}

SA and BM both provided conceptual framework for the project, data collection, and guidance for interpretation of the data, perform data analysis, participated in the laboratory investigations, performance of statically analysis and guidance for data interpretation. I also read and approved the final manuscript.

\section{Acknowledgements}

Firstly, First of all, we would like to thank our almighty God he gave us strength and health and we are deeply grateful to all the patients who took part in the project, who gave their time so generously.

We would like to acknowledge Dr. Daneal Seifu , Dr. Frank Ashell, and Dr. Wondimagegnehu Tigneh for their continuous constructive instructions as well as comments which directed us to a successful preparation of this paper.

We specifically would like to thank Dr. Frank Ashell for your provision of sample collection materials and day to day follow up. 
Our special appreciation also goes to Mrs. Aynalem Endale for your unforgettable support throughout our work.

Our last but not least gratitude also goes to Addis Ababa University College of Health Science for funding this for data collection and analysis.

\section{References}

1. Kareem R., Qassimb WJ. Ibrahim R., (2016). Assessment of psychosocial aspects of patients with rectal cancer under chemotherapy in Baghdad city. J Contemp Med Sci | Vol. 2, No. 6, pp 36-41

2. Mustafa M., Menon J., Muniandy RK., Illzam EL.,Shah MJ. Sharifa AM., (2016). Colorectal Cancer: Pathogenesis, Management and Prevention; IOSR Journal of Dental and Medical Sciences (IOSR-JDMS) e-ISSN: 2279-0853, p-ISSN: 2279-0861.Volume 15, Issue 5 Ver. IV, PP 94-100

3. Siegel R., Jemal A., (2013).Colorectal Cancer Facts \& Figures 2011-2013.National Home Office: American Cancer Society Inc. 250 Williams Street, NW, Atlanta, GA 30303-1002 (404) pp 320-3333

4. American Society of Clinical Oncology. (2016). www.asco.org | www.cancer.net | Www.conquercancerfoundation.org.

5. Jemal A., Bray F., (2011). Global cancer statistics. Cancer, J Clin; 61:69.

6. Raxitkumar J., Prateek L., Chetna J., Sergio M., Suthat L., (2013).The Association between metabolic syndrome and colorectal neoplasm. J. Clin Gastroenterology. January; 47(1): pp33-44. Doi: 10.1097/MCG

7. Simard EP, Watson M, Saraiya M, Clarke CA, Palefsky JM, Jemal A. Trends in the occurrence of high-grade anal intraepithelial neoplasia in San Francisco: 20002009. Cancer. 2013;119(19):3539-3545.Jeong Hwan K., Yun JeongL., Young-Ho K., InKyung S.,Sang Goon S., Siegel R., Miller K., Jemal A., (2015). Cancer statistics. Cancer J Clin; 65:5pp.1543 -1545 
8. Raluca P., Horatiu S., Alina C., Mihai Lucian R., Dan Lucian D., (2009).Metabolic syndrome and risk of subsequent colorectal cancer. World J Gastroenterology; 15(41): pp 5141-5148

9. Zemenfes D., Kotisso B., (2015). Colorectal and Anal Malignancies at Tukir Anbessa Specialized Hospital, Addis Ababa, Ethiopia. East and Central African Journal of Surgery; Volume 20 (2) pp $10-16$

10. Ashenafi S., (2000). the frequency of large bowel cancer as seen in Addis Ababa University, Pathology Department. Ethiop Med J. Oct; 38(4):277-82

11. Janja S., (2009). metabolic factors and Cancer risk. Department of Surgical and Perioperative Sciences Urology and Andrology, Umeå University, Umeå Sweden; No 1267, ISSN: 0346-6612, ISBN: 978-91, 7264-784-8

12. Rostom A., Dube C., Lewin G., (2007).Non-steroidal anti-inflammatory drugs and cyclooxygenase-2 inhibitors for primary prevention of colorectal cancer: a systematic review prepared for the U.S. Preventive Services Task Force. Ann Intern Med; 146(5):pp376-8

13. Silvia R., Mario R., Raffaele P., David D., Patrizia F., Fiorella G., (2014). Obesity and Colorectal cancer: Role of adipokines in tumor initiation and progression. World $J$ Gastroenterol, May 14; 20(18): 5177-5190

14. Yueh AE, Payne SN, Leystra AA, Van De Hey DR, Foley TM, Pasch CA, et al. (2016)Colon Cancer Tumor genesis Initiated by the H1047R Mutant PI3K. PLoS ONE 11(2):e0148730. https://doi.org/10.1371/journal.pone.0148730

15. Yang L, Levi E, Du J, Zhou H, Miller R, et al. (2016) Associations between Markers of Colorectal Cancer Stem Cells, Mutations, Mirna, and Clinical Characteristics of Ulcerative Colitis. Transl Med (Sunnyvale) 6: 168. doi:10.4172/2161-1025.100016

16. Nicola F, L Wilding J. Bodmer W. Genetics of colorectal cancer: hereditary aspects and overview of colorectal tumorigenesis; British Medical Bulletin 2002;64: 27-43

17. Sebastián A. G., Swiersy A, Radhakrishnan P., Branchi V., Nanduri L., Győrffy B., Betzler A., Bork U., Kahler C., Reißfelder C., . Rahbari N., Weitz J., Schölch S., LDB1 overexpression is a negative prognostic factor in colorectal cance, Oncotarget, 2016, Vol. 7, (No.51), pp: 84258-84270 
18. Giacomo P., Angelica S., Romano C., Giuseppe P., (2010). TNM Staging System of Colorectal Carcinoma. Arch Pathol Lab Med; 134: pp 837-852

19. Ayşe Neslin A., Serdar Y., Zeynep Tuğba Ö., Fatma Gökşin C., Süleyman S., Tarık Gandi C., Akın Ç., Cahit Ö., (2014). TNM and Modified Dukes staging along with the demographic characteristics of patients with colorectal carcinoma. Int J Clin Exp Med; 7(9):2828-2835

20. Johns Hopkins medicine colorectal cancer center, (2015). Staging of Colorectal Cancer; 1800 Orleans Street, Baltimore, Maryland 21287

21. Hideki U., Hidetaka M., Yoshito A., Takaya K.i, Kazutaka Y., Masahiro I., Hiroshi K., Shingo K., Yasuo O., Tadahiko M., Ryoji K., Keiichi T., Yoichi A., Kazuo H., Atsushi O., Ryo W., Keiichi I., Hideyuki S., Takahiro N., and Kenichi S., (2012). Optimal Colorectal Cancer Staging Criteria in TNM Classification. J Clin Oncol 30(13):pp15191526

22. Christian I., Okafor T., (2012). The Metabolic syndrome in Africa. Indian Journal of Endocrinal Metab. Jan-Feb; 16(1): pp 56-66.

23. Krishnan B., Ian D., Harriet F., Isabel dos-S., David A L., Liam S., (2015). BMI and risk of 22 specific cancers. National Institute for Health Research http:// dx.doi. org / 10.1016/S0140-6736(14) pp 61172-7

24. O'Neill \& O'Driscoll L., (2014). The Metabolic syndrome: a closer look at the growing epidemic and associated pathologies, Obesity review (IASO); 16: pp 1-2

25. Ji Yeon K., Suk JungY., Jung H., Hong J K., Kyun ChoY,, Chong Il S., Kyu Jeon W., B Kim BI., Choi KY., Park DI.,(2016). Different risk factors for advanced colorectal neoplasm in young adults. World J Gastroenterol ; 22(13) pp3611-3620.

26. O’Neill S. \& O'Driscoll L., (2015). metabolic syndrome: a closer look at the growing epidemic and its associated pathologies. Obesity reviews 16, pp1-12.

27. Rehana L. A., Kathryn H. S., Kristin E. A., Wayne D. R., Aaron R. F.,(2006).the Metabolic Syndrome and Risk of Incident Colorectal Cancer. American Cancer Society; 6 Jul 1; 107(1):pp28-36 DOI 10.1002/cncr.

28. Daniel T., Suzane R., Cláudio M., Cristina T., Cláudia C., João M., (2015). Metabolic syndrome and colorectal neoplasms: An ominous association. World J Gastroenterol; 21(17): pp 5320-5327 
29. Sung O. Sin-Sil Park., S. Y. Hee J., Poong-Lyul R., Jae J.K., Jong Chul R., Yoon-Ho C. (2007) Cancer Epidemiology Biomarkers. Prev; 16(8):1543-6.

30. Mehmet U., Ozge Telci C., Aytekin O., (2016). Association between Metabolic Syndrome and Cancer. Ann Nut Metab; 68: pp173-179 DOI: 10.1159/000443743

31. Belaynesh T., Molla A., Zelalem A., Nebiyu M., (2014). Metabolic syndrome among hypertensive patients. Gonder University BMC; 14: 177

32. Sebahat B., Metin U., Ayse K., Ferdane P., Abdullah O., Yasar N., Zeliha A., (2015). Absence of non-alcoholic fatty liver disease in the presence of insulin resistance is a strong predictor for colorectal carcinoma. Int J Clin Exp Med; 8(10):18601-18610

33. Slavica T. and Mahasin A. O., (2015).the double trouble of metabolic diseases: the diabetes-cancer link. Volume 26 pp3129 - 3134. DOI:10.1091/mbc.E14-11-1550

34. Bardou M., BarkunA, Martel M, Obesity and colorectal cancer Gut2013;62:933947.doi:10.1136/gutjin-2013-304701Magdalena H., Marcin D., Joanna W., Iwona K., Janusz S., (2015). Omentin - a new adipokine with many roles to play Curr. Issues Pharm. Med. Sci., Vol. 28, No.3, pp 176-180

35. Elizabeth D K., Ruzan U., Lisa B S., Edward L G., Scott M., Katja F., (2015). Adolescent body mass index and erythrocyte Sedimentation rate in relation to colorectal cancer risk. BMJ; 55(17): pp 1-7

36. Arnold m.,Pandya N.,Byrnes G., Renehan A.,Stevens G.,Ezzoti M.,Ferlay J.,Miranda J.,Romieu I.,Dikshit R.,Farman D.,Soerjomataram I.,Global burden of cancer attributable to high body- mass index ;Lancet oncol 2015;16:36 - 46

37. Bhaskaran K., Douglas I, Forbes H., dos-Santos-Silva I., A Leon D., Smeeth L.. Bodymass index and risk of 22 specifi c cancers: www.thelancet.com August 14, 2014 http://dx.doi.org/10.1016/S0140-6736(14)60892-8

38. Abdo J. , Assad E., Sahar Al K., Marie Noel Z., Alice G., Hanine H.,Anis H., Doreid O., Michel M., Jihane T., InayaH.,Pierre S., Francesco C., Giovanni T., A., Rosalyn A. J., (2016). Inflammatory bowel disease, colorectal cancer and type 2 diabetes mellitus: The links. BBA Clinical (5) pp 16-24

39. Mehmet U., Ozge Telci C., Aytekin O., (2016). Association between Metabolic Syndrome and Cancer. Ann Nut Metab; 68: pp173-179 DOI: 10.1159/000443743 
40. Susana M. C.,Olívia R., Ana M. L., Diana C. G. A. P., Artur M. S., ( 2015). Seaweeds as Preventive Agents for Cardiovascular Diseases: From Nutrients to Functional Foods. Mar. Drugs, 13, 6838-6865; doi: 10.3390/md13116838

41. Marc-Andre C., Dabelea D., Teri L. H., Rachel C. L., Amy J. S., Nicole R. S., Rachael E. V., Hong W., Robert H.,(2008).The metabolic syndrome. Endocrine Reviews; 29(7):pp777-822.

42. O’Neill \& O’Driscoll L., (2014). The Metabolic syndrome: a closer look at the growing epidemic and associated pathologies, Obesity review (IASO); 16: pp 1-2

43. Chintan J., Bhanushali L., Krishna K., Anthony K., Spiridon K., Muhammad J., Habib, Marlon D., Euni L., (2013). Association between Lifestyle Factors and Metabolic Syndrome among African Americans in the United States. Journal of Nutrition and Metabolism, http://dx.doi.org/10.1155/2013/516475 Article ID 516475, p (6).

44. AN ESTIMATED 5-YEAR PREVALENCE OF CANCER IN ETHIOPIA, (2012).GLOBOCAN (IACR)

45. Kifle et al.,.DISEASE PREVENTION AND CONTROL DIRECTORATE; NATIONAL CANCER CONTROL PLAN OF ETHIOPIA . 2016 ;1-60.

46. Heart, Lung, and Blood Institute, which is part of the National Institutes of Health (NIH), USA, publishing body is the ATP III, (2008). https://www.mdcalc.com/ framingham coronary-heart-disease-risk-score

47. Zemenfes D., Kotisso B., (2015). Colorectal and Anal Malignancies at Tukir Anbessa Specialized Hospital, Addis Ababa, Ethiopia. East and Central African Journal of Surgery; Volume 20 (2) pp $10-16$

48. Mohammed K., Maruf R., Ferdousy B., Md. Abdullah Y., Din M., Mahbuba B., Abdur Rabban T.,(2016). Clinico-demographic Characteristics of Colorectal Carcinoma in Bangladeshi Patients. Journal of Current and Advance Medical Research; 3(1):pp22-25

49. Joseph M. P., Pierre-Anthony L., Doreen Ferron-B., Patrick O. R., Derek I. M., Michael E. M.,(2016). Colorectal cancer survival in Jamaica. Annals of Medicine and Surgery; 6: pp 26-29

50. Gary M G., Stephen S L., Jeremy A L., Benjamin P J., Cecilia R S., (2010). Prevention, screening and treatment of colorectal cancer: a global and regional generalized cost effectiveness analysis. Cost Effectiveness and Resource Allocation; WHO 8:2 pp 2 - 16 
51. https://www.cdc.gov/cancer/colorectal/basic_info/screening/Pagelast reviewed: $C D C$ .August 8, 2016

52. AM El-T., (2010). Colorectal cancer and pollution.World J Gastroenterology. Jul 28; 16(28): pp 3475-3477

53. Villanueva CM., Gagniere B., Monfort C., Nieuwenhuijsen MJ., Cordier S., (2007). Sources of variability in levels and exposure to trihalomethanes. Environ Res, 103(2) pp211-220.

54. Rahman MB., Driscoll T., Cowie C., Armstrong BK., (2010). Disinfection by-products in drinking water and colorectal cancer: a meta-analysis. Int. J Epidemiology, 39(3) pp733-745

55. Carvajal-Moreno M., (2015). Metabolic Changes of Aflatoxin B1 to become an Active Carcinogen and the Control of this Toxin. Immunome Res; 11:104. doi:10.4172/17457580.10000104 .

56. Paul M. N. and Adrianne E., (1983). Rat Colon Carcinomas Associated with Aflatoxin and Marginal Vitamin A. Nutr Cancer.;5(1):pp 41-50

57. Renee W., Pascale W., Jose N., Dorice V., Fritz F., Frank H., (2016). Colorectal Cancer in African Americans. Clinical and Translational Gastroenterology; 7(7), pp185; doi:10.1038/ctg.2016.36

58. Monica M., (2015). Rising Rates of Sporadic Colorectal Cancer in Young Adult: A Possible Environmental Link. https://am.asco.org/rising-rates-sporadic colorectal cancer-young-adults-possible-environmental-link; ASCO. Chicago MAY 29.

59. Rosa H., Silva S., Eliane M., Cesário P., Maria C., Denise S., (2016). COLORECTAL CANCER: factors related to late diagnosis in users of the public health system treated at an Universitary Hospital in Curitiba Paraná State Brazil. Arq. Gastroenterology. 53(2):68-75.

60. Chyke A D.etal., Effectiveness of screening colonoscopy in reducing the risk of death from right and left colon cancer: a large community-based study. 2016 ;0:1-8. Doi: 10.1136/gutjnl-pp2016- 312712.

61. Riondino S., Roselli M., Palmirotta R., Della-Morte D., Ferroni P., Guadagni F.,(2014). Obesity and colorectal cancer: role of adipokines in tumor initiation and progression. 
World J Gastroenterol; 20: $\quad$ pp 5177-5190 [PMID: 24833848 DOI: 10.3748/WJG.V20.i18.5177]

62. Mehmet U., Ozge Telci C., Aytekin O., (2016). Association between Metabolic Syndrome and Cancer. Ann Nut Metab; 68: pp173-179 DOI: 10.1159/000443743

63. David J., Tim C., Keith G., Thomas R., Andrew G. R., Najib H., (2007). Physical Activity Before and After Diagnosis of Colorectal Cancer. Sports Med; 37 (11): pp 947960

64. Joseph M. P., Pierre-Anthony L., Doreen Ferron-B., Patrick O. R., Derek I. M., Michael E. M.,(2016). Colorectal cancer survival in Jamaica. Annals of Medicine and Surgery; 6: pp 26-29

65. Frederico A., Kunio K., Ajit V., (2016). Human risk of diseases associated with red meat intake: Analysis of current theories and proposed role for metabolic incorporation of a non-human sialic acid. Molecular Aspects of Medicine; 51 pp 16-30.

66. Kassier SM., (2016). Colon cancer and the consumption of red and processed meat: an association that is medium, rare or well done. South African Journal of Clinical Nutrition; 29(4):145-149

67. Abbasnezhad A., Alavinejad P., kadkhodae A., Isary E., Choghakhori R., (2016). Dietary Patterns among Colorectal Cancer Patients in Southwest of Iran: A Cross-Sectional Study. Gastroenterol Hepatol Open Access 4(4): p 106. DOI: 10.15406/ghoa. 2016. 04.00106

\section{Figures:}




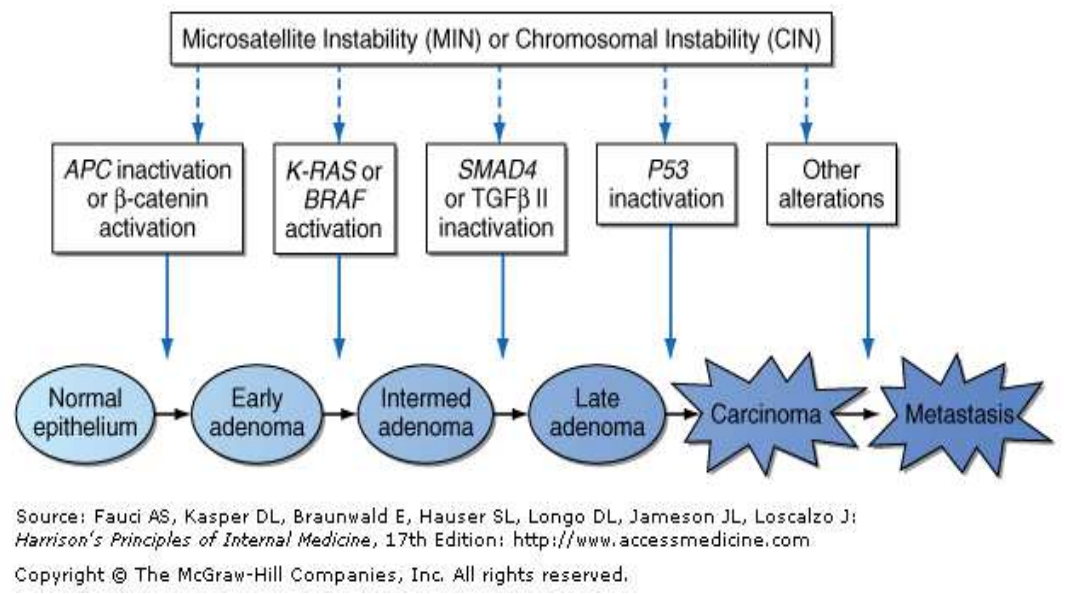

Figure- 1. Progressive somatic mutational steps in the development of colon carcinoma (Harrison's, 2008)

The accumulation of alterations in a number of different genes results in the progression from normal epithelium through adenoma to full-blown carcinoma. Genetic instability (microsatellite or chromosomal) accelerates the progression by increasing the likelihood of mutation at each step. Patients with familial polyposis are already one step into this pathway, since they inherit a germline alteration of the APC gene. TGF, transforming growth factor (Harrison's, 2008)
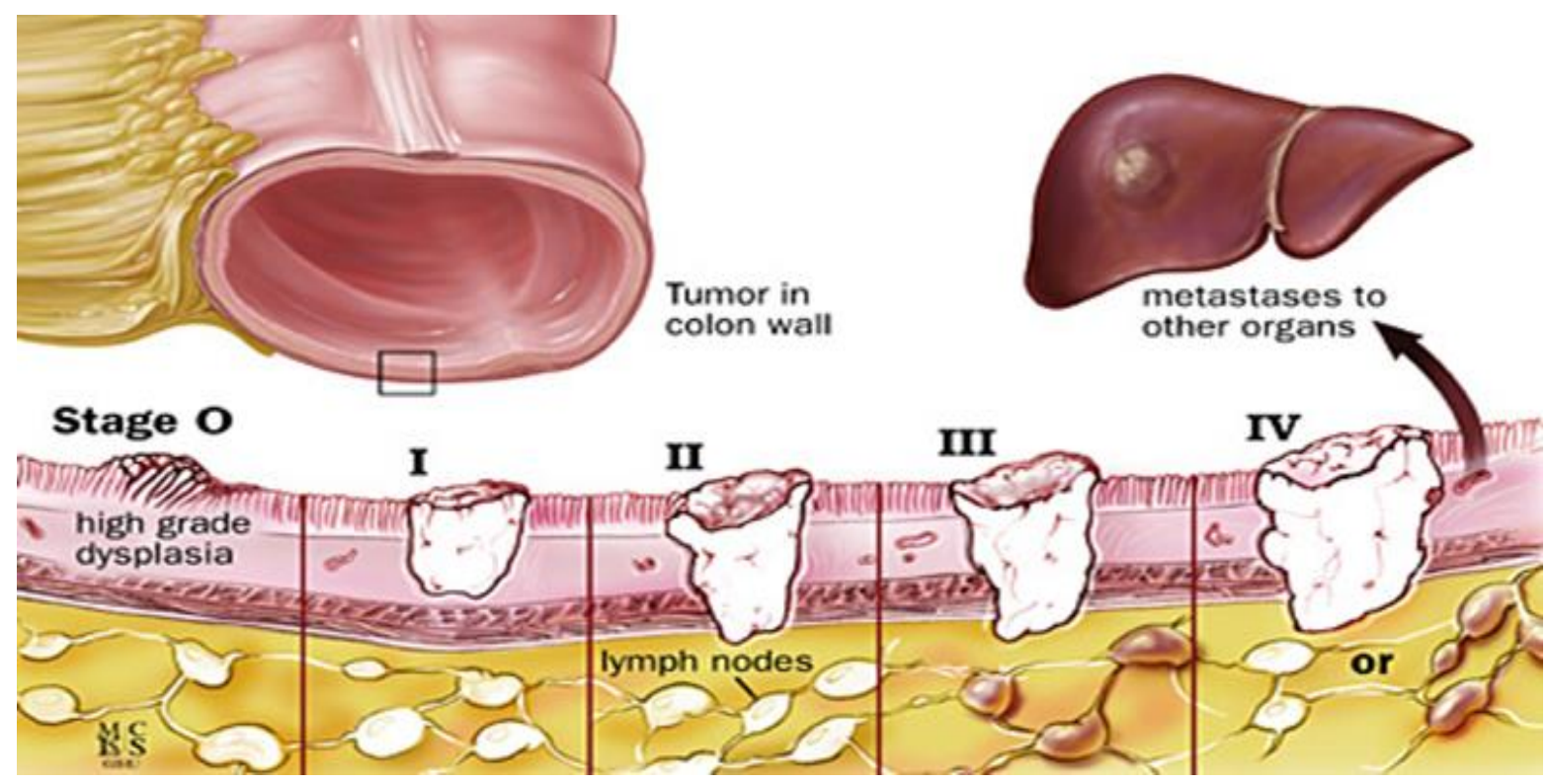

Figure-2 clinical stage of colorectal cancer (Johns Hopkins medicine colorectal cancer center,2015)

> The staging system describes whether the cancer is: in the inner lining of the colon only, embedded in the colon wall, penetrating through the colon wall, involving local lymph nodes and metastatic (spread to other organs) 


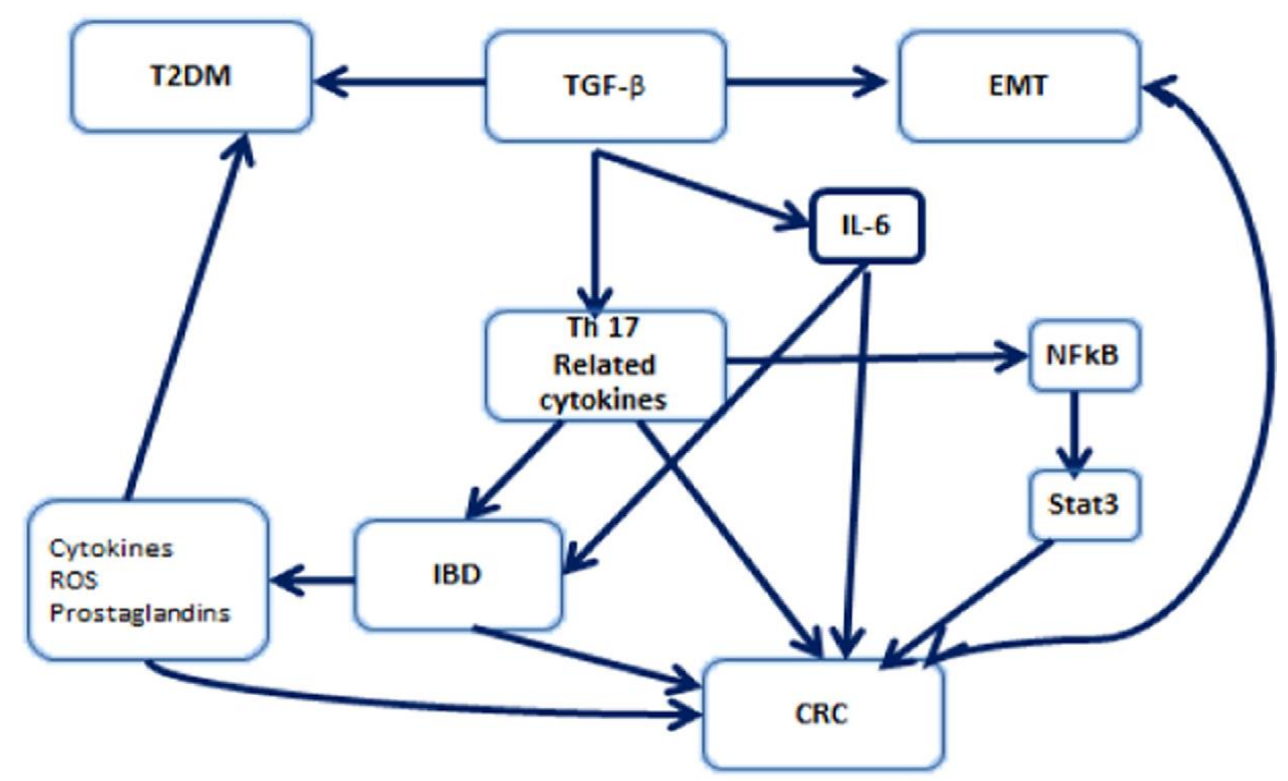

Figure-3 TGF $\beta$ creates a cancer-promoting feedback loop and is related through various pathways to IBD, CRC and T2DM. Note that blue arrows mean induction or stimulation

Key = Transforming growth factor $\beta$ (TGF- $\beta)$, nuclear factor- $k B$ (NFkB), interleukin -6 EMT=epithelial to mesenchymal transition,$C R C=$ colorectal cancer, $I B M=$ inflammatory bowel disease.

\section{Tables:}

Table -1. A comparison of TNM and Dukes' classification (Johns Hopkins Colon Cancer Center., 2015)

\begin{tabular}{|c|c|c|c|c|}
\hline \multicolumn{5}{|c|}{ TNM classification of colorectal cancer stages } \\
\hline \multicolumn{4}{|c|}{ TNM classification } & \multirow{2}{*}{$\begin{array}{l}\text { Duke's classification } \\
\text { Stages }\end{array}$} \\
\hline Stages & $\mathrm{T}$ & $\mathrm{N}$ & M & \\
\hline Stage 0 & Tis & NO & M0 & \\
\hline \multirow[t]{2}{*}{ Stage I } & $\mathrm{T} 1$ & N0 & M0 & A \\
\hline & $\mathrm{T} 2$ & N0 & M0 & B1 \\
\hline \multirow[t]{2}{*}{ Stage II } & $\mathrm{T} 3$ & No & M0 & B2 \\
\hline & $\mathrm{T} 4$ & NO & M0 & B2 \\
\hline \multirow[t]{2}{*}{ Stage III } & $\mathrm{T} 1, \mathrm{~T} 2$ & $\mathrm{~N} 1$ or $\mathrm{N} 2$ & M0 & $\mathrm{C} 1$ \\
\hline & $\mathrm{T} 3, \mathrm{~T} 4$ & $\mathrm{~N} 1$ or $\mathrm{N} 2$ & M0 & $\mathrm{C} 2$ \\
\hline
\end{tabular}


Key for TNM staging

* Primary Tumor (T)

$\mathbf{T x}$ - primary tumor cannot be assessed

T0 - no evidence of primary tumor

Tis - carcinoma in situ intra epithelial or invasion of lamina propria

T1 - tumor invades sub mucosa

T2 - tumor invades muscularis propria

T3 - tumor invades muscularis propria or in to non peritonazed pericolic or perirectal tissues

T4 - tumor directly invades other organs or stractures and /or perforates visceral peritoneum

* Regional Lymph Nodes (N)

Nx - regional lymph nodes cannot be assessed

No - no regional lymph node metastasis

$\mathbf{N 1 ~ - ~ m e t a s t a s i s ~ i n ~ o n e ~ t o ~ t h r e e ~ r e g i o n a l ~}$ lymph nodes

$\mathbf{N 2}$ - metastasis in four or more regional lymph nodes

- Distant Metastasis (M)

Mx - distant metastasis cannot be assessed

M0 - no distant metastasis

M1 - distant metastasis

Table -2: Socio-demographic characteristics of the study participants.

\begin{tabular}{lllc}
\hline Characteristics & & Frequency (N) & Percent (\%) \\
\hline AGE & $20-29$ & 6 & 7.6 \\
& $30-39$ & 20 & 25.3 \\
& $40-49$ & 25 & 31.6 \\
& $50-59$ & 8 & 10.1 \\
& $60-69$ & 14 & 17.7 \\
Region & $>70$ & 6 & 7.6 \\
& Addis Ababa & 41 & 51.9 \\
& Oromiya & 18 & 22.8 \\
& Amhara & 12 & 15.2 \\
& SNNP & 7 & 8.9 \\
\hline
\end{tabular}

SNNP- Southern Nations, Nationalities and Peoples 
Table-3. Shows the sex and ages of the patients studied. Males comprised 46 (58.2\%) of the patients studied, and females $33(41.8 \%)$. Ages ranged from 26 to 78 years in males and 20 to 78 years in females.

Table -3. Age and gender distribution of colorectal cancer patients in the study

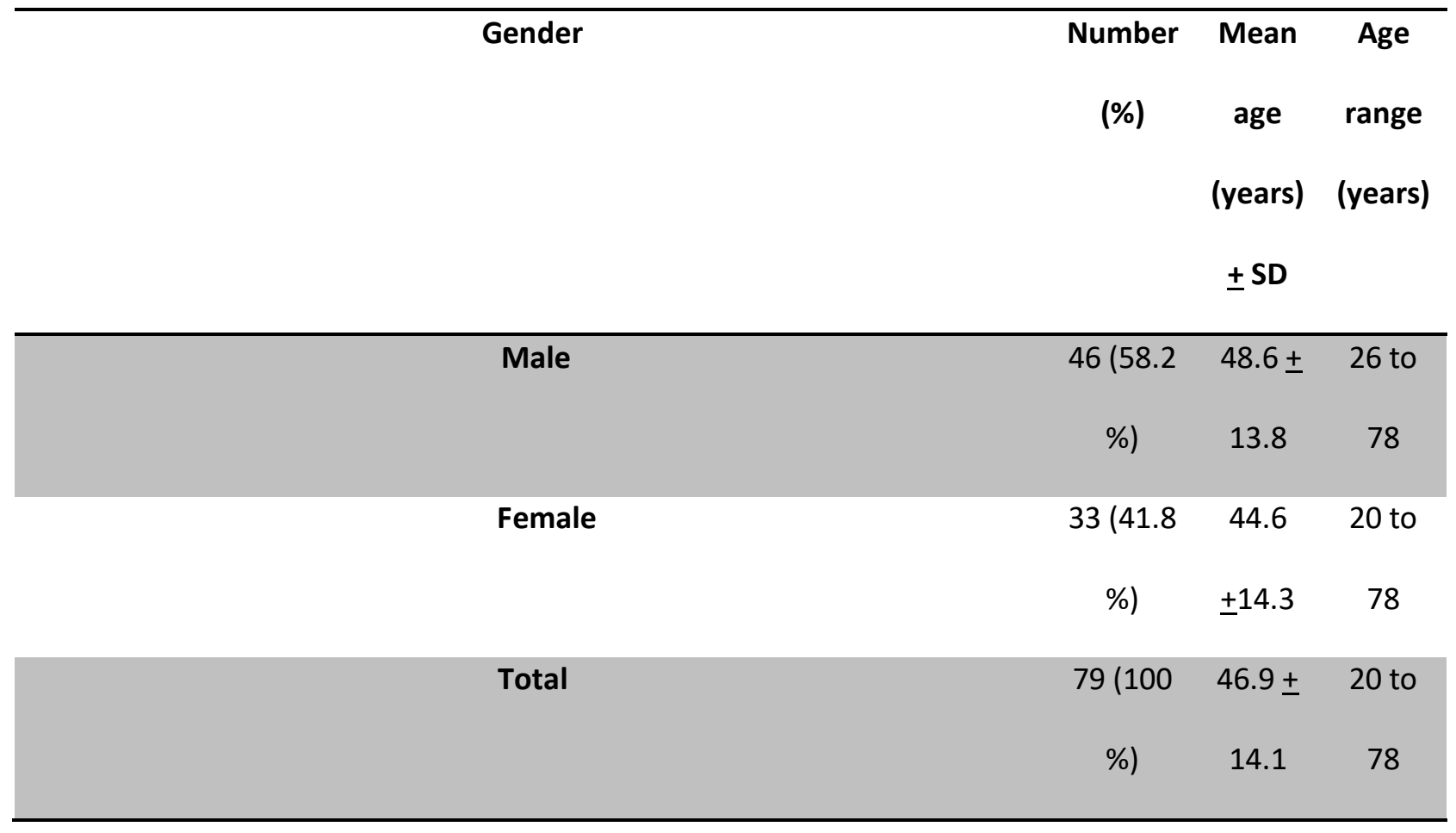

Table -4. Proportions of study patients with colon cancer and rectal cancer

\begin{tabular}{lccc}
\hline Gender & Colon CA & Rectal CA & Total \\
\hline Male & $27(58.7 \%)$ & $19(41.3 \%)$ & $46(100 \%)$ \\
Female & $15(45.5 \%)$ & $18(54.5 \%)$ & $33(100 \%)$ \\
Total & $42(53.2 \%)$ & $37(46.8 \%)$ & $79(100 \%)$ \\
\hline
\end{tabular}


Table -5. TNM stages of the different colorectal cancer patients in the study.

\begin{tabular}{cccc}
\hline $\begin{array}{c}\text { Stage of } \\
\text { colorectal Ca }\end{array}$ & Male & Gender & \\
\hline & 0 & 0 & Total \\
0 & $5(10.9 \%)$ & $5(15.2 \%)$ & $10(12.7 \%)$ \\
I & $9(19.6 \%)$ & $8(24.2 \%)$ & $17(21.5 \%)$ \\
II a & $7(15.2 \%)$ & $5(15.2 \%)$ & $12(15.2 \%)$ \\
II b & $2(4.3 \%)$ & $1(3.0 \%)$ & $3(3.8 \%)$ \\
II c & $2(4.3 \%)$ & 0 & $2(2.5 \%)$ \\
III a & $7(15.2 \%)$ & $6(18.2 \%)$ & $13(16.5 \%)$ \\
III b & $3(6.5 \%)$ & $2(6.1 \%)$ & $5(6.3 \%)$ \\
III c & $11(23.9 \%)$ & $6(18.2 \%)$ & $17(21.5 \%)$ \\
IV & & $33(100 \%)$ & $79(100 \%)$ \\
Total & $46(100 \%)$ & & \\
\hline
\end{tabular}

Table- 6. Stages of colorectal cancer in study patients according to whether they are colon cancer or rectal cancer

\begin{tabular}{cccc}
\hline Stage of cancer & \multicolumn{2}{l}{ Type of colorectal cancer } & \\
\cline { 2 - 4 } & $\underline{\text { Colon cancer }}$ & $\underline{\text { Rectal cancer }}$ & $\underline{\text { Total }}$ \\
I & $4(9.5 \%)$ & $6(16.2 \%)$ & $10(12.7 \%)$ \\
II & $20(47.6 \%)$ & $12(32.4 \%)$ & $32(40.5 \%)$ \\
III & $10(23.8 \%)$ & $10(27 \%)$ & $20(25.3 \%)$ \\
Total & $\underline{8(19 \%)}$ & $\underline{9(24.3 \%)}$ & $\underline{17(21.5 \%)}$ \\
\hline
\end{tabular}

Table -7. Body Mass Index (BMI) of colorectal cancer patients in the study

For definitions of BMI categories, see Materials and Methods.

\begin{tabular}{cccccc}
\hline \multicolumn{5}{c}{ Nender } & \multicolumn{5}{c}{} \\
\hline BMI & $<18.5$ & $18.5-24.9$ & $25-29.9$ & $\geq 30$ & Total \\
& Underweight & Normal BMI & Overweight & Obese & \\
\hline
\end{tabular}




\begin{tabular}{cccccc}
\hline M & $4(8.7 \%)$ & $21(45.7 \%)$ & $19(41.3 \%)$ & $2(4.3 \%)$ & $46(100 \%)$ \\
F & $8(24.2 \%)$ & $11(33.3 \%)$ & $13(39.4 \%)$ & $1(3.0 \%)$ & $33(100 \%)$ \\
M + F & $12(15.2 \%)$ & $32(40.5 \%)$ & $32(40.5 \%)$ & $3(3.8 \%)$ & $79(100 \%)$ \\
\hline
\end{tabular}

Table -8. Prevalence of parameters of Metabolic Syndrome among colorectal cancer patients in the study

\begin{tabular}{|c|c|c|c|}
\hline \multirow[t]{2}{*}{ Parameter } & \multicolumn{3}{|c|}{ Number (\%) of patients } \\
\hline & Male & Female & Total \\
\hline $\begin{array}{c}\frac{\text { Abnormal waist }}{\text { circumference }} \\
M>102 \mathrm{~cm} \\
F>88 \mathrm{~cm}\end{array}$ & $12(15 \%)$ & $9(11.4 \%)$ & $21(26.6 \%)$ \\
\hline $\begin{array}{c}\text { Elevated blood pressure } \\
\text { Sys } \geq 130 \mathrm{mmHg} \\
\text { Dias } \geq 85 \mathrm{mmHg}\end{array}$ & $31(29.2 \%)$ & $5(6.3 \%)$ & $36(45.6 \%)$ \\
\hline$\frac{\text { Fasting blood glucose }}{>100 \mathrm{mg} / \mathrm{dL}}$ & 30 (38 \%) & $23(29 \%)$ & $53(67.1 \%)$ \\
\hline $\begin{array}{l}\text { Serum HDL. } \\
M<40 \mathrm{mg} / \mathrm{dl} \\
F<50 \mathrm{mg} / \mathrm{dl}\end{array}$ & $30(38 \%)$ & $24(30.4 \%)$ & $54(63.3 \%)$ \\
\hline$\frac{\text { Serum triglyceride }}{>150 \mathrm{mg} / \mathrm{dL}}$ & $30(38 \%)$ & $15(18.9 \%)$ & 45 (57 \%) \\
\hline $\begin{array}{c}\text { Patients with metabolic } \\
\text { syndrome } \\
\text { ( } \geq 3 \text { parameters) }\end{array}$ & $10(12.7 \%)$ & $13(16.5 \%)$ & $23(29 \%)$ \\
\hline
\end{tabular}

Table -9. Framingham Score (Mean +/- SD) of smokers and non-smokers in the study

\begin{tabular}{rc}
\hline Participants & Framingham score (mean \pm SD) \\
\hline Non-smoker & $8.2 \pm 6.6$ \\
Smoker & $14 \pm 11.4$ \\
\hline
\end{tabular}

Table-11. Number and percentage of colorectal cancer patients with elevated serum LDL cholesterol 


\begin{tabular}{ccc}
\hline Gender & LDL $\geq 130$ & LDL $\leq 130$ \\
\hline M & $18(39.9 \%)$ & $28(60.9 \%)$ \\
F & $12(36.4 \%)$ & $21(63.6 \%)$ \\
Total & $30(38 \%)$ & $49(62 \%)$ \\
\hline
\end{tabular}

Table-10: Framingham Risk Scores of smokers among the colorectal cancer patients in the study, and risk if the patients were non-smokers.

\begin{tabular}{|c|c|c|c|c|c|c|c|c|c|c|}
\hline $\begin{array}{l}\stackrel{0}{2} \\
\text { 으 }\end{array}$ & 品 & 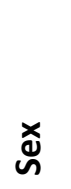 & 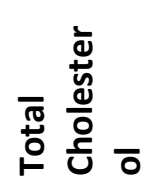 & $\overline{\mathbf{I}}$ & $\begin{array}{l}\frac{.0}{0} \\
\stackrel{5}{n} \\
\text { जे }\end{array}$ & 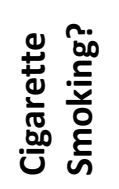 & 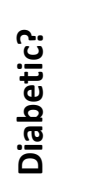 & z点 & $\begin{array}{l}\% \text { risk } \\
\text { as } \\
\text { smoker }\end{array}$ & $\begin{array}{l}\text { \% risk as a } \\
\text { non- } \\
\text { smoker }\end{array}$ \\
\hline BD 3 & 60 & $\mathrm{M}$ & 180 & 59 & 120 & Yes & No & No & 17.5 & 9.5 \\
\hline NG7 & 52 & $M$ & 104 & 35 & 100 & Yes & No & No & 7.4 & 3.9 \\
\hline AB16 & 38 & $\mathrm{M}$ & 128 & 55 & 140 & Yes & No & No & 4.5 & 2.4 \\
\hline MT34 & 65 & $\mathrm{M}$ & 170 & 38 & 140 & Yes & No & No & 37.4 & 21.6 \\
\hline ZM55 & 60 & $\mathrm{~F}$ & 148 & 49 & 110 & Yes & No & No & 5.7 & 3.4 \\
\hline AA66 & 65 & $M$ & 135 & 40 & 100 & Yes & No & No & 16.4 & 8.9 \\
\hline MG67 & 40 & $M$ & 180 & 43 & 110 & Yes & No & No & 6.1 & 3.2 \\
\hline GM68 & 72 & $\mathrm{M}$ & 184 & 52 & 100 & Yes & No & No & 23.8 & 13.2 \\
\hline
\end{tabular}




\section{Figures}

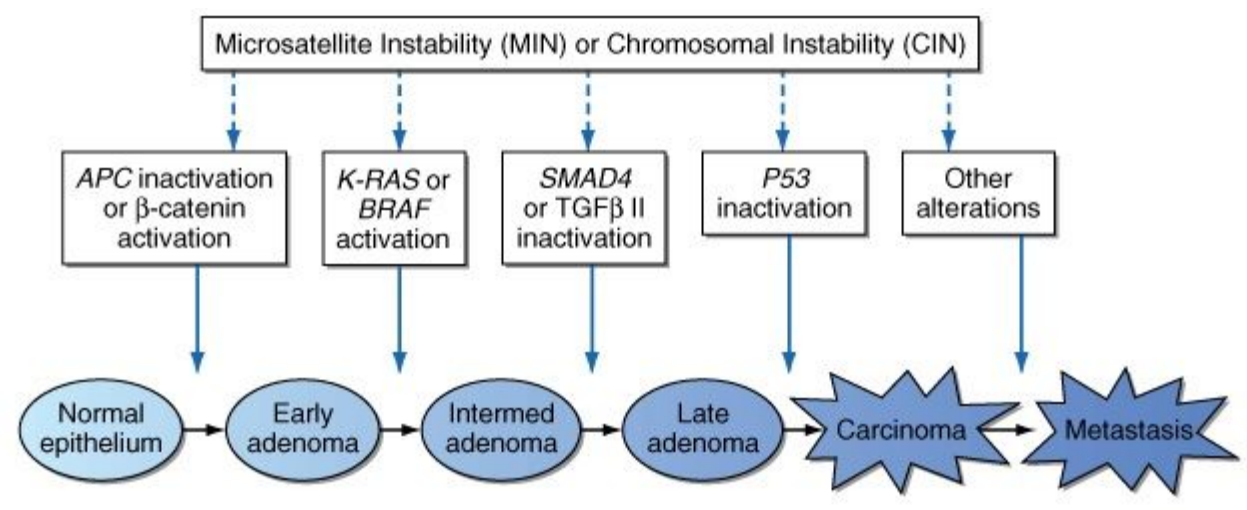

Source: Fauci AS, Kasper DL, Braunwald E, Hauser SL, Longo DL, Jameson JL, Loscalzo J: Harrison's Principles of Internal Medicine, 17th Edition: http://www. accessmedicine.com

Copyright 9 The McGraw-Hill Companies, Inc. All rights reserved.

\section{Figure 1}

Progressive somatic mutational steps in the development of colon carcinoma (Harrison's, 2008) The accumulation of alterations in a number of different genes results in the progression from normal epithelium through adenoma to full-blown carcinoma. Genetic instability (microsatellite or chromosomal) accelerates the progression by increasing the likelihood of mutation at each step. Patients with familial polyposis are already one step into this pathway, since they inherit a germline alteration of the APC gene. TGF, transforming growth factor (Harrison's, 2008)
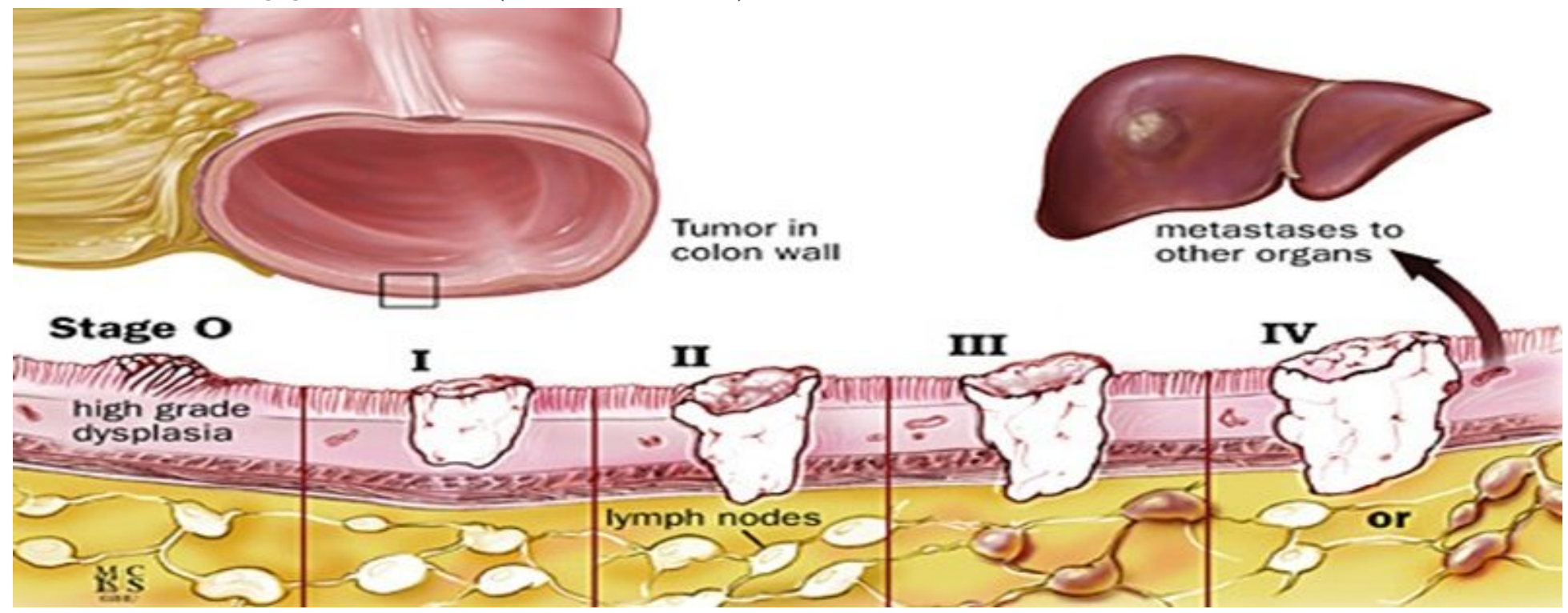

\section{Figure 2}

clinical stage of colorectal cancer (Johns Hopkins medicine colorectal cancer center,2015) $>$ The staging system describes whether the cancer is: in the inner lining of the colon only, embedded in the colon wall, penetrating through the colon wall, involving local lymph nodes and metastatic (spread to other organs) 


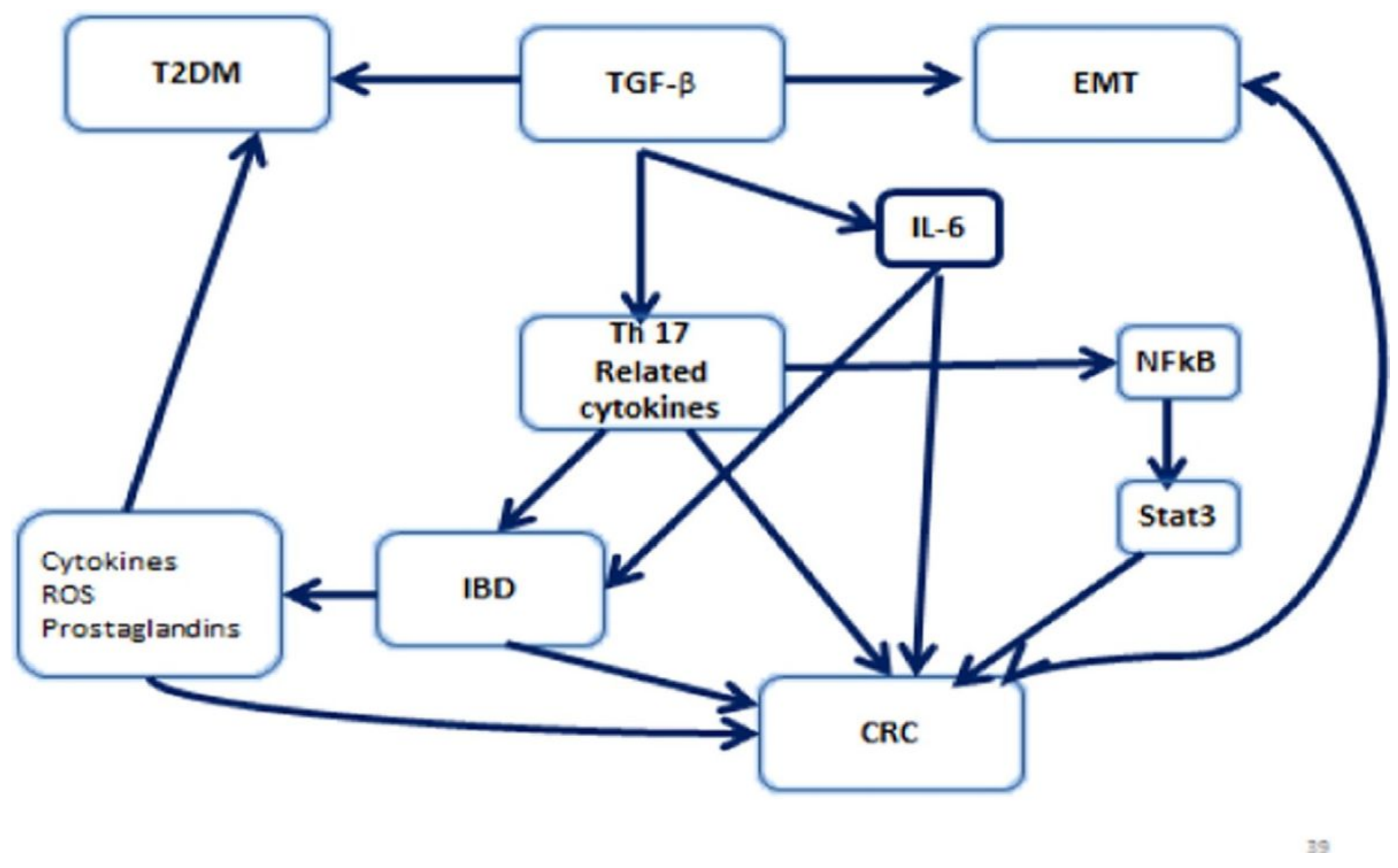

Figure 3

TGF $\beta$ creates a cancer-promoting feedback loop and is related through various pathways to IBD, CRC and T2DM. Note that blue arrows mean induction or stimulation Key $=$ Transforming growth factor $\beta$ (TGF- $\beta$ ), nuclear factor- $\mathrm{kB}$ (NFkB), interleukin $-6 \mathrm{EMT}=$ epithelial to mesenchymal transition ,CRC = colorectal cancer, IBM = inflammatory bowel disease. 